\title{
Prueba de difusión pulmonar de monóxido de carbono con técnica de una sola respiración (DL,COsb). Recomendaciones y procedimiento
}

\author{
Pulmonary carbon monoxide diffusing capacity with the single \\ breath technique (DL,COsb). Procedure and recommendations
}

\author{
Juan Carlos Vázquez-García,, Laura Gochicoa-Rangel,* Rodrigo Francisco Del Río-Hidalgo,* Silvia Cid-Juárez, \\ Mónica Silva-Cerón,* José Luis Miguel-Reyes, ${ }^{*}$ Rosaura Benítez-Pérez,* Rogelio Pérez-Padilla, ${ }^{*}$ Luis Torre-Bouscoulet*
}

*Instituto Nacional de Enfermedades Respiratorias Ismael Cosío Villegas, Ciudad de México.

\begin{abstract}
RESUMEN. La difusión pulmonar de monóxido de carbono (DL,CO, por sus siglas en inglés), también referida como factor de transferencia de monóxido de carbono (TL,CO), se usa para evaluar la transferencia de oxígeno desde el espacio alveolar hasta la hemoglobina de los eritrocitos contenidos en los capilares pulmonares. En este manuscrito abordamos los principios fisiológicos de la prueba de DL,CO con la técnica de una sola respiración (DL,COsb) y emitimos recomendaciones, tanto para su ejecución como para su interpretación. El procedimiento se apega al estándar vigente publicado en 2005 por la Sociedad Americana del Tórax y la Sociedad Respiratoria Europea.
\end{abstract}

Palabras clave: Difusión pulmonar de monóxido de carbono, DL,COsb, pruebas de función pulmonar.

\section{INTRODUCCIÓN}

La difusión pulmonar de monóxido de carbono (DL,CO, por sus siglas en inglés), también referida como factor de transferencia de monóxido de carbono (TL,CO), se usa para evaluar la transferencia de oxígeno desde el espacio alveolar hasta la hemoglobina de los eritrocitos contenidos en los capilares pulmonares. ${ }^{1,2}$ Es la principal prueba para la evaluación del intercambio de gases a nivel pulmonar. Existen varias técnicas de medición, pero la más común por su disponibilidad, facilidad y mayor

\section{Correspondencia:}

Dr. Juan Carlos Vázquez-García

Instituto Nacional de Enfermedades Respiratorias Ismael Cosío Villegas.

Calzada de Tlalpan Núm. 4502,

Colonia Sección XVI, 14080, Ciudad de México.

Correo electrónico: drjcvazquez@gmail.com

\begin{abstract}
Pulmonary carbon monoxide diffusing capacity (DL,CO), also referred as lung transfer factor of carbon monoxide $(T L, C O)$ is used to evaluate the transfer of oxygen from the alveolar space to the hemoglobin erythrocytes contained in the pulmonary capillaries. In this manuscript we address the physiological principles of testing $\mathrm{DL}, \mathrm{CO}$ with the single breath technique (DL,COsb) and issue recommendations for their implementation as well as for interpretation. The procedure is compliant with the current standards published in 2005 by the American Thoracic Society (ATS) and the European Respiratory Society (ERS).
\end{abstract}

Keywords: Pulmonary carbon monoxide diffusing capacity, DL,CO, pulmonary function tests.

estandarización es la técnica de una sola respiración (DL,COsb). ${ }^{3}$ Además, durante la medición de $\mathrm{DL}, \mathrm{CO}$ se agrega un gas inerte, helio $(\mathrm{He})$, metano $(\mathrm{CH} 3)$ o neón $(\mathrm{Ne})$, que sirve para calcular la ventilación alveolar $\left(\mathrm{V}_{\mathrm{A}}\right)$ y medir los volúmenes pulmonares (TLC y RV) por medio de la dilución del gas. ${ }^{4}$

Cuando la DL,COsb se realiza bajo condiciones estandarizadas y un adecuado control de calidad detecta cambios en la función pulmonar, incluso menores al $10 \% .{ }^{5}$ Un valor disminuido de DL,CO se puede observar en las principales enfermedades pulmonares crónicas como son la enfermedad pulmonar obstructiva crónica (EPOC) y la enfermedad pulmonar intersticial difusa (EPID). Los valores de DL,CO correlacionan directamente con el grado de enfisema y de inflamación o fibrosis, respectivamente. ${ }^{6-10} \mathrm{La} \mathrm{DL}, \mathrm{CO}$ también se puede afectar en las enfermedades vasculares pulmonares o por anormalidades de la hemoglobina, anemia o valores elevados de carboxihemoglobina $(\mathrm{COHb}) .{ }^{11-13}$ 


\section{Indicaciones de la prueba}

En general, la principal indicación de la prueba de $\mathrm{DL}, \mathrm{CO}$ es la evaluación diagnóstica y el seguimiento de las enfermedades del parénquima pulmonar. Las indicaciones precisas son: $:^{1,3,6-10}$

1. Evaluación y seguimiento de pacientes con EPID.

2. Evaluación y seguimiento de pacientes con EPOC.

3. Diagnóstico diferencial entre bronquitis crónica, enfisema y asma.

4. Evaluación de la afección pulmonar en enfermedades sistémicas.

5. Evaluación de enfermedades vasculares pulmonares: vasculitis, hipertensión pulmonar y tromboembolia pulmonar crónica.

6. Predicción de la desaturación arterial durante el ejercicio en algunos pacientes con enfermedad pulmonar.

7. Evaluación y cuantificación del impedimento o incapacidad asociados con enfermedades del parénquima pulmonar.

8. Evaluación preoperatoria en: resección pulmonar, cirugía de reducción de volumen y trasplante pulmonar.

9. Evaluación de los efectos pulmonares de los agentes quimioterapéuticos y otros medicamentos que se conoce causan daño pulmonar, así como de radioterapia.

10. Evaluación de hemorragia pulmonar.

11. Evaluación de algunas enfermedades infecciosas pulmonares difusas (ej.: neumonía por Pneumocystis).

12. Diagnóstico oportuno y seguimiento en los programas de vigilancia respiratoria en medicina ocupacional, en especial en sujetos expuestos a polvos inorgánicos.

\section{Contraindicaciones ${ }^{1,2,14,15}$}

Absolutas:

1. Hipoxemia grave $\left(\mathrm{SpO}_{2}<75\right)$. En este caso su realización se puede evaluar de acuerdo con la altitud, siempre bajo supervisión médica.

2. Niveles de carboxihemoglobina elevados $(\mathrm{COHb}>10$ a $15 \%)$.

Relativas:

1. Confusión o pobre coordinación muscular que impida realizar la maniobra adecuada.

2. Enfermedad cardiovascular aguda o descompensada (infarto, insuficiencia cardíaca, enfermedad cerebrovascular).

3. Neumotórax en los últimos tres meses.

4. Riesgo de sangrado por hemoptisis o aneurismas.

5. Cirugía en el último mes (tórax, abdomen, ojos, oído).
6. Infecciones respiratorias agudas en las últimas dos semanas (influenza, catarro común).

7. Tuberculosis pulmonar activa.

8. Embarazo avanzado o complicado.

9. Pacientes con traqueostomía o sondas pleurales.

10. Pacientes que no puedan suspender oxígeno suplementario por lo menos durante 30 minutos.

11. Pacientes con capacidad vital (VC) o capacidad vital forzada (FVC) menor a los volúmenes mínimos requeridos por el equipo.

\section{Requerimientos del equipo de $\mathrm{DL}, \mathrm{CO}^{3,5,15}$}

Los equipos de $\mathrm{DL}, \mathrm{CO}$ deben reunir las recomendaciones técnicas internacionales emitidas por la Sociedad Americana del Tórax y la Sociedad Europea Respiratoria (ATS/ ERS 2005), ${ }^{3}$ con los siguientes requerimientos mínimos recomendados para las mediciones de volumen y el analizador de gases:

1. El equipo debe cumplir con los requerimientos de medición de volumen establecidos por la ATS/ERS para espirometría. ${ }^{16}$

2. El analizador de gases de monóxido de carbono (CO) y de gas trazador deben cumplir respuesta lineal desde una concentración cero hasta la concentración completa del gas de prueba. El error en la respuesta lineal del analizador no debe sobrepasar más del $0.5 \%$ en la escala completa. Por ejemplo, si la concentración de $\mathrm{CO}$ en el gas de prueba es de $0.300 \%$, el máximo error en cualquier dilución del gas debe ser $\pm 0.0015 \%$.

3. El analizador de gases debe ser estable durante toda la prueba, con un mínimo desplazamiento en cero y en ganancia. El desplazamiento permitido debe de ser de $\pm 0.001 \%$ sobre la concentración absoluta de CO y de $\pm 0.5 \%$ en la escala completa del gas trazador.

4. La presencia de bióxido de carbono $\left(\mathrm{CO}_{2}\right)$ y vapor de $\mathrm{H}_{2} \mathrm{O}$ no debe interferir con el analizador de gases. Si es así, el equipo debe remover estos gases antes de que la muestra pase por el analizador o que el equipo haga los ajustes en la medición de los gases de acuerdo con la concentración de $\mathrm{CO}_{2}$ y vapor de $\mathrm{H}_{2} \mathrm{O}$ presentes.

5. La resistencia del circuito debe ser $<1.5 \mathrm{CmH}_{2} \mathrm{O} \cdot \mathrm{L}^{-1} \cdot \mathrm{S}^{-1} \mathrm{a}$ un flujo de $6 \mathrm{~L} \cdot \mathrm{s}^{-1}$, si el tanque de gas de prueba usa un regulador de demanda de flujo, la presión inspiratoria máxima a través del circuito y de la válvula debe de ser $<10 \mathrm{cmH}_{2} \mathrm{O}$.

6. El cronómetro del dispositivo debe tener una exactitud de $1 \%$ (100 ms sobre 10 segundos).

7. El equipo debe permitir seleccionar la técnica para medir el tiempo de apnea escrita por Jones-Mead. ${ }^{17}$

8. Capacidad para medir el volumen de espacio muerto anatómico $\left(V_{D}\right)$ para gas inspirado y el volumen de 
muestreo de gas alveolar. El $V_{D}$ incluye la válvula, el filtro y la pieza bucal y no debe exceder $350 \mathrm{~mL}$. Sin embargo, se puede requerir de $V_{D}$ más pequeño para niños o pacientes con baja VC.

9. El sistema debe estar libre de fugas.

\section{Otro equipamiento y consumibles}

1. Computadora e impresora, de acuerdo con requerimientos del dispositivo.

2. Escalas para medición de peso, estatura y cinta métrica para medición de extensión de brazos, cuando se requiera.

3. Termómetros ambientales con una precisión de $1^{\circ} \mathrm{C}$.

4. Boquilla con filtro en línea desechable con eficiencia de $>99 \%$ para filtración de virus, bacterias y micobacterias; espacio muerto $<100 \mathrm{~mL}$ y resistencia menor $1.5 \mathrm{cmH}_{2} \mathrm{O}$ a un flujo de $6 \mathrm{~L} / \mathrm{s}$.

5. Aditamentos para control de infecciones:
a. Acceso a lavado de manos y gel desinfectante.
b. Guantes desechables.
c. Cubreboca quirúrgico para protección general y cuando se requiera cubreboca N95 que requiere fuga menor al $10 \%$ y una eficiencia de filtrado de $>95 \%$ a un flujo de $50 \mathrm{~L} / \mathrm{min}$.
d. Anteojos de protección.

\section{Preparación del equipo antes de la prueba ${ }^{3,5,15,18}$}

1. Los sensores de flujo deben limpiarse siguiendo las recomendaciones del fabricante y estar libres de partículas que obstruyan el sensor.

2. Todos los componentes (mangueras, sensores, conectores, etc.) deben ser ensamblados de acuerdo con las instrucciones del fabricante.

3. Se debe encender el equipo y dar tiempo suficiente para el calentamiento del mismo.

4. En caso necesario debe revisarse para fugas.

5. Si el dispositivo no cuenta con termómetro interno, requiere medición de temperatura ambiental antes de la calibración y antes de la prueba.

6. El dispositivo debe estar codificado a la altitud o presión barométrica y humedad relativa promedio del sitio donde se realiza la prueba.

7. El dispositivo debe de estar codificado para medición del tiempo de apnea por el método de Jones-Mead. ${ }^{17}$

8. En caso de no existir ecuaciones de referencia validadas para población local, se recomienda que el dispositivo esté codificado con valores normales del estudio NHANES.

9. El sensor de flujo o volumen debe de calibrarse de acuerdo con las recomendaciones establecidas por la ATS/ERS. ${ }^{16}$

\section{Instrucciones para el paciente antes de la prueba ${ }^{1,3,5,15}$}

1. El paciente debe evitar fumar por lo menos dos horas antes de la prueba.

2. Evitar el uso de prendas restrictivas de tórax como chalecos, corsés o ropa muy ajustada.

3. El paciente no tiene que suspender cualquier otra medicación de base.

4. No se requiere de ayuno para la prueba, pero se recomienda alimentación ligera.

5. Evitar ejercicio intenso cuatro horas antes.

6. Se recomienda aplicar un cuestionario breve de historia médica que incluya:

a. Historia de tabaquismo, actual o pasado, número total de años de fumador y promedio diario de cigarros por día.

b. Historia de exposición laboral a humos o polvos, número total de años de exposición y promedio de horas por día.

c. Historia de síntomas respiratorios: disnea, sibilancias, tos y expectoración.

d. Historia de enfermedades o traumas toracopulmonares.

e. Contraindicaciones de la prueba: enfermedad cardiovascular aguda, infecciones respiratorias agudas o activas (catarro común, influenza, tuberculosis, etc.), embarazo avanzado o complicado.

\section{Preparación del paciente para la prueba ${ }^{3,15}$}

1. El técnico que realiza la prueba recibe y se presenta con el paciente.

2. El paciente debe descansar sentado durante al menos 10 minutos, mientras el técnico completa la preparación.

3. Confirmar el nombre completo del paciente y que coincida con la solicitud médica y con el número de registro, si aplica.

4. El técnico explica al paciente el objetivo de la prueba.

5. Revisar las contraindicaciones de la prueba. En presencia de una contraindicación, el técnico no debe realizar la prueba, a menos que el médico tratante lo solicite por escrito o lo autorice el director médico del laboratorio.

6. Registrar el consumo de tabaco, el ejercicio físico intenso antes de la prueba y el uso de broncodilatadores.

7. La estatura se mide en centímetros, con el individuo sin zapatos, en posición completamente erguida, talones juntos y mirando al frente. Para la medición de estatura es recomendable el uso de estadímetros.

8. En los pacientes que no puedan mantenerse de pie o sufran deformidad de caja torácica, se puede usar la extensión de los brazos como una estimación de la 
estatura. Se solicita al paciente que extienda al máximo los brazos en direcciones opuestas. Se mide la extensión entre los extremos de los dedos medios de cada mano. La estatura se estima para hombres como extensión de brazos/1.03 y para mujeres extensión de brazos/1.01.

9. El peso se mide en una báscula y se registra en kilogramos, en unidades cerradas al $0.5 \mathrm{~kg}$ más cercano. El peso no es utilizado en la mayoría de las ecuaciones de referencia para calcular valores normales, pero es útil para fines de interpretación.

10. La edad se registra en años cumplidos al día de la prueba.

11. La prueba debe realizarse con el sujeto sentado en una silla cómoda, sin ruedas, con respaldo y apoyo para los brazos.

12. Se instruye al paciente o sujeto sobre el uso adecuado de la boquilla; ésta debe sujetarse con los dientes, sin morderse, sellarla con los labios alrededor de la boquilla y evitar meter la lengua en el orificio de la boquilla.

13. El técnico explica la maniobra haciendo énfasis en respiración en volumen corriente, maniobra de volumen de reserva espiratoria, capacidad vital inspiratoria, apnea de 10 segundos, seguida de espiración mayor a 4 segundos.

\section{PROCEDIMIENTO $3,5,15$}

1. Debe colocarse al sujeto en la posición correcta, sentado, con los dos pies apoyados en el suelo, con el tronco erguido y la cabeza ligeramente elevada; esto debe mantenerse durante toda la maniobra.

2. El individuo se coloca adecuadamente la boquilla. Siempre debe usarse una boquilla nueva con filtro con cada paciente.

3. Se coloca la pinza nasal.

4. Se realizan de dos a tres respiraciones en volumen corriente.

5. Desde el nivel de capacidad funcional residual (al final de una espiración normal), se indica que realice espiración máxima, hasta alcanzar una meseta de un segundo al llegar a volumen residual (maniobra de volumen de reserva espiratoria). Al llegar a volumen residual se activan las válvulas.

6. Se estimula al paciente a realizar inspiración máxima y rápida (menos de 3 segundos), la cual debe alcanzar más del $90 \%$ de su VC con una tolerancia de hasta $85 \%$ (mínimo requerido por ATS/ERS 2005). ${ }^{7}$

7. Se debe mantener el período de apnea durante 10 segundos, teniendo precaución de que no haya fugas o maniobra de Valsalva durante la apnea.

8. Se instruye al sujeto o paciente a realizar una espiración máxima, pero de manera lenta y relajada, mayor a 4 segundos.
9. En caso de una maniobra fallida se deben repetir las instrucciones y la demostración si es necesario.

10. El tiempo entre maniobras debe ser de al menos 4 minutos.

11. Se requiere completar un mínimo de dos esfuerzos que reúnan criterios de aceptabilidad.

12. Se debe revisar el criterio de repetibilidad.

13. Se pueden realizar hasta cinco maniobras para obtener un mínimo de dos que sean aceptables y repetibles.

El espirograma de la maniobra de DL,COsb puede observarse en la figura 1.

\section{REVISIÓN DE RESULTADOS}

\section{Criterios de aceptabilidad ${ }^{3,5,15}$}

1. La maniobra de ERV (volumen de reserva espiratorio) debe mostrar meseta (mínimo de un segundo) que compruebe que se ha alcanzado el nivel de RV (volumen residual).

2. El volumen inspiratorio es al menos de $90 \%$ (con un mínimo tolerado de $85 \%$ ) de la VC o FVC.

3. El tiempo de apnea recomendado es de $10 \pm 1$ segundo (estándar de $10 \pm 2$ ).

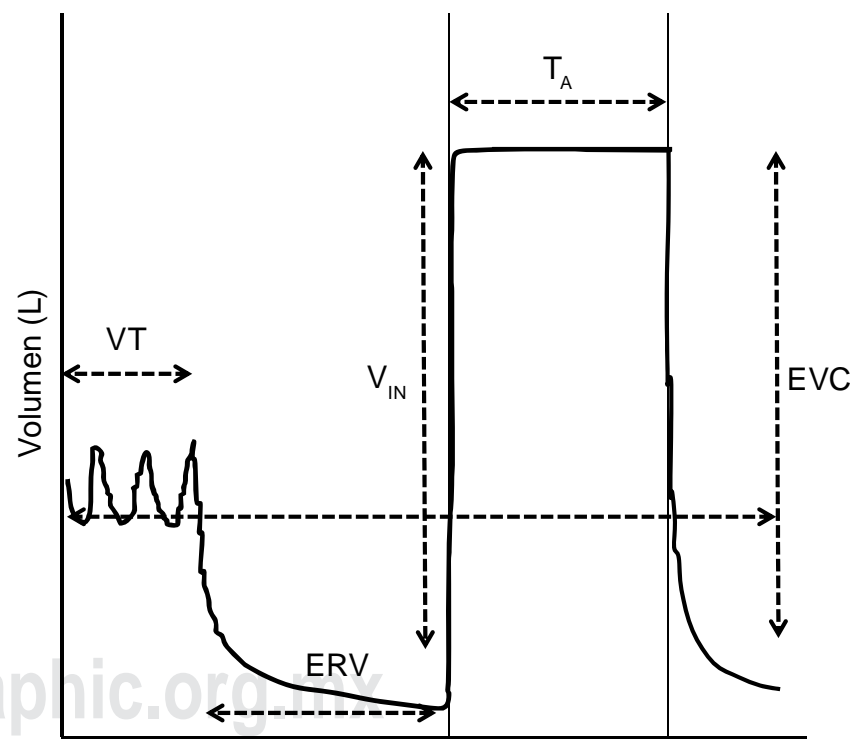

Tiempo (s)

Figura 1: Maniobra de DL,CO. La maniobra inicia con tres respiraciones en volumen corriente (VT) seguidas de una maniobra de volumen de reserva espiratoria (ERV). Al llegar a volumen residual el sujeto debe completar una capacidad vital inspiratoria que determina el volumen inspiratorio de la maniobra $\left(\mathrm{V}_{\mathrm{IN}}\right)$. Posterior a ello se realiza la apnea de 10 segundos seguida de espiración no forzada durante al menos 4 segundos (EVC). El tiempo de apnea (TA) se calcula por el método de Jones-Mead que incluyen el $70 \%$ del tiempo inspiratorio hasta la mitad del tiempo a la mitad del volumen de muestra. 
4. El espirograma está libre de fugas o maniobras de Müller o Valsalva.

5. El tiempo espiratorio es de por lo menos 4 segundos.

6. El tiempo para obtener el volumen de gas de muestra es menor a 3 segundos.

7. El volumen de lavado $\left(\mathrm{V}_{\mathrm{D}}\right)$ es de 750 a $1,000 \mathrm{~mL}$ (recomendado $750 \mathrm{~mL}$ ).

8. El volumen de muestreo $\left(\mathrm{V}_{\mathrm{s}}\right)$ es de 500 a $1,000 \mathrm{~mL}$ (recomendado de $500 \mathrm{~mL}$ ).

\section{Valoración de repetibilidad $3,5,15$}

1. El criterio de repetibilidad debe ser utilizado durante la realización de la prueba sólo para decidir si se necesitan más de dos maniobras aceptables de DL,CO.

2. Cinco maniobras es un límite tolerado para alcanzar la repetibilidad de la prueba en la mayoría de los sujetos. No se deben realizar más de cinco esfuerzos ya que un número mayor incrementa la cantidad de carboxihemoglobina, lo que puede afectar la medición.

3. El criterio de repetibilidad no se usa para excluir maniobras del reporte o excluir sujetos de un estudio.

4. La mayoría de las personas son capaces de alcanzar una repetibilidad para $\mathrm{DL}, \mathrm{CO}$ menor de tres unidades. Sin embargo, el estándar incluye 10\% del valor absoluto.

5. Los resultados finales de la repetibilidad de la $\mathrm{DL}, \mathrm{CO}$ deben ser utilizados para fines de interpretación.

6. Las pruebas no aceptables o con pobre repetibilidad deben ser evaluadas a discreción del profesional responsable de la interpretación.

\section{Informe de la DL,CO}

El contenido del informe de la DL,CO depende de los parámetros contenidos en el software. Es recomendable que incluya la información suficiente para que se pueda evaluar la calidad de la prueba, así como para la interpretación. Se recomienda incluir los siguientes componentes:

1. Nombre completo.

2. Parámetros generales (edad, género, peso y estatura).

3. Origen de los valores de referencia.

4. DL,CO medido sin corrección, como valor final de $\mathrm{DL}, \mathrm{CO}$ se considera el promedio de dos maniobras aceptables.

5. Porcentaje del predicho de la DL,CO.

6. Valores absolutos en BTPS (condiciones corporales de presión, temperatura y saturación de vapor de agua) y predichos de: $\mathrm{V}_{I^{\prime}}, V_{A^{\prime}}, T L C_{S B}$ (capacidad pulmonar total mediante maniobra de única respiración) y $\mathrm{RV}_{S B}$ (volumen residual mediante maniobra de única respiración).
7. La relación de $\mathrm{DL}, \mathrm{CO} / \mathrm{N}_{\mathrm{A}}$, también conocida como $\mathrm{KCO}$ (VA se reporta como BTPS).

8. Cualquier ajuste (ej.: $\mathrm{Hb}, \mathrm{COHb}$ o $\mathrm{PiO}_{2}$ ) debe ser reportado por separado junto con los valores utilizados para el cálculo.

9. Gráficos que incluyan espirograma y concentraciones de los gases.

\section{Anotaciones especiales y limitaciones del procedimiento}

1. Las concentraciones de $\mathrm{COHb}$ pueden alterar la prueba de DLCO. ${ }^{12}$

2. La DL,CO se incrementa con las grandes altitudes por menor competencia de oxígeno debido a menor $\mathrm{PiO}_{2} \cdot{ }^{19}$

3. La DL,CO puede variar por posición corporal, puede aumentar en posición de supino. ${ }^{20}$

4. La maniobra de Valsalva puede disminuir la DL,CO debido a que disminuye la cantidad de sangre en los capilares. ${ }^{3,5}$

5. Se ha descrito un incremento de la DL,CO durante el embarazo (primer trimestre) no consistentemente encontrado en otros estudios. ${ }^{21-23}$

6. Se ha informado una variación diurna (caída de 1.2\%/h de las 9:30 a.m. a las 5:50 p.m.). ${ }^{24}$

7. Existe un cambio de hasta $13 \%$ durante los ciclos menstruales. ${ }^{25}$ El valor más alto de DL,CO se da justo antes de la menstruación y el valor menor al tercer día de la menstruación.

8. Puede haber una reducción de hasta el $15 \%$ del valor de DL,CO a los 90 minutos de haber ingerido alcohol., ${ }^{1,26}$

9. El tabaquismo altera los resultados de la prueba. ${ }^{3}$

\section{Ajustes en el valor de DL,CO}

1. Debe hacerse un ajuste por la concentración de $\mathrm{Hb}$ cuando sea posible ${ }^{27}$ (tablas 1 y 2).

2. Existe un pequeño incremento en la $\mathrm{COHb}$ (alrededor de $0.7 \%$ por inhalación del gas de prueba) durante la prueba.

3. No se requiere ajustar por $\mathrm{COHb}$, excepto cuando se sospeche elevada, como en fumadores, para mejorar la interpretación (tabla 3).

4. $\mathrm{DL}, \mathrm{CO}$ ajustado por $\mathrm{COHb}=\mathrm{DL}, \mathrm{CO}$ medido $(1 \%+\% \mathrm{COHb} / 100) .^{3}$

5. Ajuste por altitud (tablas 4 y 5 ):

a. $\quad \mathrm{DL}, \mathrm{CO}$ ajustado por altitud $=\mathrm{DL}, \mathrm{CO}$ medido $\mathrm{X}[1$ $\left.+0.0031\left(\mathrm{PiO}_{2}-150\right)\right]$, donde $\mathrm{PiO}_{2}$ estimada $=0.21$ (presión barométrica -47), o se pueden ajustar los valores predichos.

b. DL,CO predicho por altitud $=\mathrm{DL}, \mathrm{CO}$ predicho / $\left[1+0.0031\left(\mathrm{PIO}_{2}-150\right)\right]$. 
Tabla 1: Valores predichos de DL,CO para hombres adultos ajustados para hemoglobina.

\begin{tabular}{|c|c|c|c|c|c|c|c|c|c|c|c|c|c|c|c|c|c|c|c|c|c|}
\hline \multirow{2}{*}{$\begin{array}{l}\text { DL, } \\
\text { Co } \\
\% \mathrm{p}\end{array}$} & \multicolumn{21}{|c|}{ Valor de hemoglobina } \\
\hline & 5 & 6 & 7 & 8 & 9 & 10 & 11 & 12 & 13 & 14 & 15 & 16 & 17 & 18 & 19 & 20 & 21 & 22 & 23 & 24 & 25 \\
\hline 5 & 2.8 & 3.1 & 3.5 & 3.7 & 4.0 & 4.2 & 4.4 & 4.6 & 4.8 & 4.9 & 5.1 & 5.2 & 5.3 & 5.4 & 5.5 & 5.6 & 5.7 & 5.8 & 5.9 & 6.0 & 6.0 \\
\hline 6 & 3.4 & 3.8 & 4.1 & 4.5 & 4.8 & 5.0 & 5.3 & 5.5 & 5.7 & 5.9 & 6.1 & 6.2 & 6.4 & 6.5 & 6.6 & 6.8 & 6.9 & 7.0 & 7.1 & 7.2 & 7.2 \\
\hline 7 & 3.9 & 4.4 & 4.8 & 5.2 & 5.6 & 5.9 & 6.2 & 6.4 & 6.7 & 6.9 & 7.1 & 7.3 & 7.4 & 7.6 & 7.7 & 7.9 & 8.0 & 8.1 & 8.2 & 8.3 & 8.4 \\
\hline 8 & 4.5 & 5.0 & 5.5 & 6.0 & 6.4 & 6.7 & 7.0 & 7.3 & 7.6 & 7.9 & 8.1 & 8.3 & 8.5 & 8.7 & 8.8 & 9.0 & 9.1 & 9.3 & 9.4 & 9.5 & 9.7 \\
\hline 9 & 5.0 & 5.7 & 6.2 & 6.7 & 7.2 & 7.6 & 7.9 & 8.3 & 8.6 & 8.8 & 91 & 9 & 9.6 & 9.8 & 9.9 & 10.1 & 10.3 & 10.4 & 10.6 & 10.7 & 10.9 \\
\hline 12 & 6.7 & 7.5 & 8.3 & 9.0 & 9.6 & 10.1 & 10.6 & 11.0 & 11.4 & 11.8 & 12.1 & 12.4 & 12.7 & 13.0 & 13.3 & 13.5 & 13.7 & 13.9 & 14.1 & 14.3 & 14.5 \\
\hline 13 & 7.3 & 8.2 & 9.0 & 9.7 & 10.3 & 10.9 & 11.5 & 11.9 & 12.4 & 12.8 & 13.1 & 13.5 & 13.8 & 14.1 & 14.4 & 14.6 & 14.9 & 15.1 & 15.3 & 15.5 & 15.7 \\
\hline 14 & 7.8 & 8.8 & 9.7 & \begin{tabular}{|l|}
10.5 \\
\end{tabular} & 11.1 & 11.8 & 12.3 & 12.9 & 13.3 & 13.8 & \begin{tabular}{|l|}
14.2 \\
\end{tabular} & 14.5 & 14.9 & 15.2 & 15.5 & 15.8 & 16.0 & \begin{tabular}{|l|}
16.3 \\
\end{tabular} & 16.5 & 16.7 & 16.9 \\
\hline 15 & 8.4 & 9.4 & 10.4 & \begin{tabular}{|l}
11.2 \\
\end{tabular} & 11.9 & 12.6 & \begin{tabular}{|l}
13.2 \\
\end{tabular} & 13.8 & 14.3 & 14.7 & \begin{tabular}{|l}
15.2 \\
\end{tabular} & 15.6 & 15.9 & 16.3 & 16.6 & 16.9 & 17.2 & \begin{tabular}{|l|}
17.4 \\
\end{tabular} & 17.7 & 17.9 & \begin{tabular}{|l|}
18.1 \\
\end{tabular} \\
\hline 16 & 8.9 & 10.1 & 11.1 & \begin{tabular}{|l}
11.9 \\
\end{tabular} & 12.7 & 13.5 & 14.1 & 14.7 & 15.2 & 15.7 & 16.2 & 16.6 & 17.0 & 17.3 & 17.7 & 18.0 & 18.3 & 18.6 & 18.8 & 19.1 & 19.3 \\
\hline 17 & 9.5 & 10.7 & 11.7 & \begin{tabular}{|l|}
12.7 \\
\end{tabular} & 13.5 & 14.3 & 15.0 & 15.6 & 16.2 & 16.7 & \begin{tabular}{|l|l}
17.2 \\
\end{tabular} & 17.6 & 18.0 & 18.4 & 18.8 & 19.1 & 19.4 & \begin{tabular}{|l|}
19.7 \\
\end{tabular} & 20.0 & 20.3 & 20.5 \\
\hline 22 & 12.3 & 13.8 & 15.2 & \begin{tabular}{|l}
16.4 \\
\end{tabular} & 17.5 & 18.5 & 19.4 & 20.2 & 20.9 & 21.6 & \begin{tabular}{|l}
22.2 \\
\end{tabular} & 22.8 & 23.4 & 23.9 & 24.3 & 24.8 & 25.2 & \begin{tabular}{|l}
25.5 \\
\end{tabular} & 25.9 & 26.2 & 26.5 \\
\hline 23 & 12.8 & 14.5 & 15.9 & \begin{tabular}{|l}
17.2 \\
\end{tabular} & 18.3 & 19.3 & 20.3 & 21.1 & 21.9 & 22.6 & \begin{tabular}{|l}
23.3 \\
\end{tabular} & 23.9 & 24.4 & 24.9 & 25.4 & 25.9 & 26.3 & \begin{tabular}{|l|}
26.7 \\
\end{tabular} & 27.1 & 27.4 & \begin{tabular}{|l|}
27.8 \\
\end{tabular} \\
\hline 24 & 13.4 & 15.1 & 16.6 & \begin{tabular}{|l|}
17.9 \\
\end{tabular} & 19.1 & 20.2 & 21.1 & 22.0 & 22.8 & 23.6 & 24.3 & 24.9 & 25.5 & 26.0 & 26.5 & 27.0 & 27.4 & 27.9 & 28.2 & 28.6 & 29.0 \\
\hline 25 & 14.0 & 15.7 & 17.3 & 18. & 19.9 & 21.0 & 22.0 & 23.0 & 23.8 & 24.6 & 25 & 2 & 26.5 & 27.1 & 27 & 28.1 & 28.6 & 29.0 & .4 & 8 & .2 \\
\hline 26 & 14.5 & 16.4 & 18.0 & 19.4 & 20.7 & 21.9 & 22.9 & 23.9 & 24.7 & 25.5 & 26.3 & 27.0 & 27.6 & 28.2 & 28.7 & 29.3 & 29.7 & 30.2 & 30.6 & 31.0 & 31.4 \\
\hline 27 & 15.1 & 17.0 & 18.7 & \begin{tabular}{|l}
20.2 \\
\end{tabular} & 21.5 & 22.7 & 23.8 & 24.8 & 25.7 & 26.5 & \begin{tabular}{|l}
27.3 \\
\end{tabular} & 28.0 & 28.7 & 29.3 & 29.8 & 30.4 & 30.9 & \begin{tabular}{|l}
31.3 \\
\end{tabular} & 31.8 & 32.2 & 32.6 \\
\hline 28 & 15.6 & 17.6 & 19.3 & \begin{tabular}{|l|}
20.9 \\
\end{tabular} & 22.3 & 23.5 & 24.7 & 25.7 & 26.6 & 27.5 & 28 & 2 & 29.7 & 30.4 & 0 & 31.5 & 32.0 & 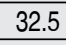 & 0 & 3.4 & \begin{tabular}{|l|}
33.8 \\
\end{tabular} \\
\hline 29 & 16.2 & 18.2 & 20.0 & \begin{tabular}{|l}
21.6 \\
\end{tabular} & 23.1 & 24.4 & 25.6 & 26.6 & 27.6 & 28.5 & 29.3 & 30.1 & 30.8 & 31.4 & 32.1 & 32.6 & 33.2 & 33.7 & 34.1 & 34.6 & 35.0 \\
\hline 30 & 16.8 & 18.9 & 20.7 & \begin{tabular}{|l}
22.4 \\
\end{tabular} & 23.9 & 25.2 & 26.4 & 27.5 & 28.6 & 29.5 & 30.3 & 3 & 31.9 & 32.5 & 33.2 & 33.8 & 34.3 & 34.8 & 35.3 & 35.8 & 36.2 \\
\hline 31 & 17.3 & 19.5 & 21.4 & \begin{tabular}{|l|}
23.1 \\
\end{tabular} & 24.7 & 26.1 & 27.3 & 28.5 & 29.5 & 30.5 & \begin{tabular}{|l|}
31.3 \\
\end{tabular} & 32.2 & 32.9 & 33.6 & 34.3 & 34.9 & 35.4 & 36.0 & 36.5 & 37.0 & 37.4 \\
\hline 32 & 17.9 & 20.1 & 22.1 & 23.9 & 25.5 & 26.9 & 28.2 & 29.4 & 30.5 & 31 & 32. & 33.2 & 34.0 & 34.7 & 35.4 & 36.0 & 36.6 & 37.1 & 37.7 & 38.2 & 38.6 \\
\hline 41 & 22.9 & 25.8 & 28.3 & $\begin{array}{l}30.6 \\
\end{array}$ & 32.6 & 34.5 & 36.1 & 37.6 & 39.0 & 40.3 & 41.5 & 42.5 & 43.5 & 44.5 & 45.3 & 46.1 & 46.9 & \begin{tabular}{|l|}
47.6 \\
\end{tabular} & 48.3 & 48.9 & 49.5 \\
\hline 42 & 23.5 & 26.4 & 29.0 & 31.4 & 33.4 & 35.3 & 37.0 & 38.6 & 40.0 & 41.3 & \begin{tabular}{|l}
42.5 \\
\end{tabular} & 43.6 & 44.6 & 45.5 & 46.4 & 47.3 & 48.0 & \begin{tabular}{|l}
48.8 \\
\end{tabular} & 49.4 & 50.1 & 50.7 \\
\hline 43 & 24.0 & 27.0 & 29.7 & 32.1 & 34.2 & 36.2 & 37. & 39.5 & 40.9 & 42.3 & 43 & 44 & 45.7 & 46.6 & 47.5 & 48.4 & 49.2 & 49.9 & 50.6 & 51.3 & 51.9 \\
\hline 44 & 24.6 & 27.7 & 30.4 & \begin{tabular}{|l}
32.8 \\
\end{tabular} & 35.0 & 37.0 & 38.8 & 40.4 & 41.9 & & 44.5 & 45.6 & 46.7 & 47.7 & 48.6 & 49.5 & 50.3 & 51.1 & 1.8 & 52.5 & 53.1 \\
\hline 45 & 25.1 & 28.3 & 31.1 & \begin{tabular}{|l}
33.6 \\
\end{tabular} & 35.8 & 37.8 & 39.7 & 41.3 & 42.8 & 44.2 & \begin{tabular}{|l}
45.5 \\
\end{tabular} & 46.7 & 47.8 & 48.8 & 49.7 & 50.6 & 51.5 & \begin{tabular}{|l}
52.2 \\
\end{tabular} & 53.0 & 53.7 & 54.3 \\
\hline 46 & 25.7 & 28.9 & 31.8 & 34.3 & 36.6 & 38.7 & 40.5 & 42.2 & 43.8 & 45.2 & 46.5 & 47.7 & 48.8 & 49.9 & 50.8 & 51.8 & 52.6 & 53.4 & 54.1 & 54.8 & 55.5 \\
\hline 47 & 26.2 & 29.6 & 32.5 & \begin{tabular}{|l|}
35.1 \\
\end{tabular} & 37.4 & 39.5 & 41.4 & 43.2 & 44.7 & 46.2 & \begin{tabular}{|l|}
47.5 \\
\end{tabular} & 48.8 & 49.9 & 51.0 & 52.0 & 52.9 & 53.7 & \begin{tabular}{|l|}
54.6 \\
\end{tabular} & 55.3 & 56.0 & \begin{tabular}{|l|}
56.7 \\
\end{tabular} \\
\hline 48 & 26.8 & 30.2 & 33.2 & 35.8 & 38.2 & 40.4 & 42.3 & 44.1 & 45.7 & 47.2 & \begin{tabular}{|l|}
48.5 \\
\end{tabular} & 49.8 & 51.0 & 52.0 & 53.1 & 54.0 & 54.9 & \begin{tabular}{|l|}
55.7 \\
\end{tabular} & 56.5 & 57.2 & 57.9 \\
\hline 49 & 27.4 & 30.8 & 33.9 & 36.6 & 39.0 & 41.2 & 43.2 & 45.0 & 46.6 & 48.2 & 49.5 & 50.8 & 52.0 & 53.1 & 54.2 & 55.1 & 56.0 & 56.9 & 57.7 & 58.4 & 59.1 \\
\hline 50 & 27.9 & 31.4 & 34.6 & \begin{tabular}{|l|l}
37.3 \\
\end{tabular} & 39.8 & 42.0 & 44.1 & 45.9 & 47.6 & 49.1 & 50.6 & 51.9 & 53.1 & 54.2 & 55.3 & 56.3 & 57.2 & 58.0 & 58.9 & 59.6 & 60.3 \\
\hline 51 & 28.5 & 32.1 & 35.2 & \begin{tabular}{|l|}
38.1 \\
\end{tabular} & 40.6 & 42.9 & 44.9 & 46.8 & 48.5 & 50.1 & 51.6 & 52.9 & 54.1 & 55.3 & 56.4 & 57.4 & 58.3 & 59.2 & 60.0 & 60.8 & 61.5 \\
\hline 52 & 29.0 & 32.7 & 35.9 & \begin{tabular}{|l|}
38.8 \\
\end{tabular} & 41.4 & 43.7 & 45.8 & 47.7 & 49.5 & 51.1 & \begin{tabular}{|l|}
52.6 \\
\end{tabular} & 53.9 & 55.2 & 56.4 & 57.5 & 58.5 & 59.5 & \begin{tabular}{|l|}
60.4 \\
\end{tabular} & 61.2 & 62.0 & 62.7 \\
\hline 53 & 29.6 & 33.3 & 36.6 & \begin{tabular}{|l|}
39.6 \\
\end{tabular} & 42.2 & 44.6 & 46.7 & 48.7 & 50.4 & 52.1 & \begin{tabular}{|l}
53.6 \\
\end{tabular} & 55.0 & 56.3 & 57.5 & 58.6 & 59.6 & 60.6 & \begin{tabular}{|l|l}
61.5 \\
\end{tabular} & 62.4 & 63.2 & 64.0 \\
\hline 54 & 30.2 & 34.0 & 37.3 & \begin{tabular}{|l}
40.3 \\
\end{tabular} & 43.0 & 45.4 & 47.6 & 49.6 & 51.4 & 53.1 & 54.6 & 56.0 & 57.3 & 58.6 & 59.7 & 60.8 & 61.7 & 62.7 & 63.6 & 64.4 & 65.2 \\
\hline 55 & 30.7 & 34.6 & 38.0 & 41.1 & 43.8 & 46.2 & 48.5 & 50.5 & 52.3 & 54.0 & 55.6 & 57.1 & 58.4 & 59.6 & 60.8 & 61.9 & 62.9 & 63.8 & 64.7 & 65.6 & 66.4 \\
\hline 56 & 31.3 & 35.2 & 38.7 & \begin{tabular}{|l|l}
41.8 \\
\end{tabular} & 44.6 & 47.1 & 49.3 & 51.4 & 53.3 & 55.0 & \begin{tabular}{|l}
56.6 \\
\end{tabular} & 58.1 & 59.5 & 60.7 & 61.9 & 63.0 & 64.0 & 65.0 & 65.9 & 66.8 & 67.6 \\
\hline 57 & 31.8 & 35.8 & 39.4 & 42.5 & 45.4 & 47.9 & 50.2 & 52.3 & 54.3 & 56.0 & \begin{tabular}{|l|}
57.6 \\
\end{tabular} & 59.1 & 60.5 & 61.8 & 63.0 & 64.1 & 65.2 & \begin{tabular}{|l}
66.2 \\
\end{tabular} & 67.1 & 68.0 & 68.8 \\
\hline 58 & 32.4 & 36.5 & 40.1 & \begin{tabular}{|l|}
43.3 \\
\end{tabular} & 46.2 & 48.8 & 51.1 & 53.2 & 55.2 & 57.0 & \begin{tabular}{|l|}
58.6 \\
\end{tabular} & 60.2 & 61.6 & 62.9 & 64.1 & 65.3 & 66.3 & \begin{tabular}{|l}
67.3 \\
\end{tabular} & 68.3 & 69.2 & \begin{tabular}{|l|}
70.0 \\
\end{tabular} \\
\hline 59 & 33.0 & 37.1 & 40.8 & 44.0 & 47.0 & 49.6 & 52.0 & 54.2 & 56.2 & 58.0 & \begin{tabular}{|l|}
59.7 \\
\end{tabular} & 61.2 & 62.6 & 64.0 & 65.2 & 66.4 & 67.5 & \begin{tabular}{|l|}
68.5 \\
\end{tabular} & 69.4 & 70.3 & 71.2 \\
\hline 60 & 33.5 & 37.7 & 41.5 & \begin{tabular}{|l|l}
44.8 \\
\end{tabular} & 47.8 & 50.4 & 52.9 & 55.1 & 57.1 & 59.0 & 60.7 & 62.2 & 63.7 & 65.1 & 66.3 & 67.5 & 68.6 & 69.6 & 70.6 & 71.5 & 72.4 \\
\hline
\end{tabular}


Tabla 2: Valores predichos de DL,CO para mujeres y niños (< 15 años) ajustados para hemoglobina.

\begin{tabular}{|c|c|c|c|c|c|c|c|c|c|c|c|c|c|c|c|c|c|c|c|c|c|}
\hline \multirow{2}{*}{$\begin{array}{l}\text { DL, } \\
\text { CO } \\
\% \text { p }\end{array}$} & \multicolumn{21}{|c|}{ Valor de hemoglobina } \\
\hline & 5 & 6 & 7 & 8 & 9 & 10 & 11 & 12 & 13 & 14 & 15 & 16 & 17 & 18 & 19 & 20 & 21 & 22 & 23 & 24 & 25 \\
\hline 5 & 2.2 & 2.5 & 2.8 & 3.0 & 3.2 & 3.4 & 3.5 & 3.7 & 3.8 & 3.9 & 4.0 & 4.1 & 4.2 & 4.3 & 4.4 & 4.5 & 4.6 & 4.6 & 4.7 & 4.8 & 4.8 \\
\hline 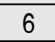 & 2.8 & 3.1 & 3.5 & 37 & 40 & 4.2 & 44 & 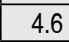 & 48 & 4.9 & 51 & 50 & 5.3 & 5.4 & 5 & 56 & 7 & 5 & 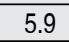 & 6.0 & 6.0 \\
\hline 1 & 3.4 & 3.8 & 4.1 & 4.5 & 4.8 & 5.0 & 5.3 & 5.5 & 5.7 & 5.9 & 6.1 & 6.2 & 6.4 & 6.5 & 6.6 & 6.8 & 6.9 & 70 & 7.1 & 7.2 & 7.2 \\
\hline 8 & 3.9 & 4.4 & 4.8 & 5.2 & 5.6 & 5.9 & 6.2 & 6.4 & 6.7 & 6.9 & 7.1 & 7.3 & 7.4 & 7.6 & \begin{tabular}{ll|}
7.7 \\
\end{tabular} & 7.9 & 8.0 & 8.1 & 8.2 & 8.3 & 8.4 \\
\hline 9 & 4.5 & 5.0 & 5.5 & 6.0 & 6.4 & 6.7 & 7.0 & 7.3 & 7.6 & 7.9 & 8.1 & 8.3 & 8.5 & 8.7 & 8.8 & 9.0 & 9.1 & 9.3 & 9.4 & 9.5 & 9.7 \\
\hline 10 & 5.0 & 5.7 & 6.2 & 6.7 & 7.2 & 7.6 & 7.9 & & 8.6 & 8.8 & 91 & 93 & 9.6 & 9.8 & 9.9 & \begin{tabular}{|l|}
10.1 \\
\end{tabular} & 10.3 & 10.4 & 10.6 & 10.7 & 10.9 \\
\hline 11 & 5.6 & 6.3 & 6.9 & 7.5 & 8.0 & 8.4 & 8.8 & 9.2 & 9.5 & 9.8 & 10.1 & 10.4 & 10.6 & 10.8 & 11.1 & \begin{tabular}{|l|}
11.3 \\
\end{tabular} & 11.4 & 1.6 & 1.8 & 11.9 & 12.1 \\
\hline 12 & 6.1 & 6.9 & 7.6 & 8.2 & 8.8 & 9.2 & 9.7 & 10.1 & 10.5 & 10.8 & 11.1 & 11.4 & 11.7 & 11.9 & 12.2 & \begin{tabular}{|c|}
12.4 \\
\end{tabular} & 12.6 & 2.8 & 2.9 & 13.1 & 13.3 \\
\hline 13 & 6.7 & 7.5 & 8.3 & 9.0 & 9.6 & 10.1 & 10.6 & 11.0 & 11.4 & 11.8 & 12.1 & 12.4 & 12.7 & 13.0 & 13.3 & \begin{tabular}{|l|}
13.5 \\
\end{tabular} & 13.7 & 3.9 & 4.1 & 4.3 & $\overline{4.5}$ \\
\hline 14 & 7.3 & 8.2 & 9.0 & 9.7 & 10.3 & 10.9 & 11.5 & 11.9 & 12.4 & 12.8 & 131 & 13.5 & 13.8 & 14.1 & 14.4 & \begin{tabular}{|l|}
14.6 \\
\end{tabular} & 14.9 & 5.1 & 15.3 & 5.5 & 15.7 \\
\hline 15 & 7.8 & 8.8 & 9.7 & 10.5 & 11.1 & 11.8 & 12.3 & 12.9 & 13.3 & 13.8 & 14.2 & 14.5 & 14.9 & 15.2 & 15.5 & \begin{tabular}{|c|}
15.8 \\
\end{tabular} & 16.0 & 6.3 & 6.5 & 6.7 & 16.9 \\
\hline 16 & 8.4 & 9.4 & 10.4 & 11.2 & 11.9 & 12.6 & 13.2 & 13.8 & 14.3 & 14.7 & 15.2 & 15.6 & 15.9 & 16.3 & 16.6 & \begin{tabular}{|l|}
16.9 \\
\end{tabular} & 17.2 & 17.4 & 17.7 & 17.9 & 18.1 \\
\hline 17 & 8.9 & 10.1 & \begin{tabular}{|l|l}
11.1 \\
\end{tabular} & 11.9 & 12.7 & 13.5 & 14.1 & 14.7 & 15.2 & 15.7 & & & 17.0 & & \begin{tabular}{|l|}
17.7 \\
\end{tabular} & \begin{tabular}{|l|}
18.0 \\
\end{tabular} & & & & & 100 \\
\hline 18 & 9.5 & \begin{tabular}{|l}
10.7 \\
\end{tabular} & \begin{tabular}{|l|l}
11.7 \\
\end{tabular} & 12.7 & 13.5 & 14.3 & 15.0 & 15.6 & 16.2 & 16.7 & 4 & 17.6 & 18.0 & 18.4 & \begin{tabular}{|c|}
18.8 \\
\end{tabular} & \begin{tabular}{|l|}
19.1 \\
\end{tabular} & 9.4 & .7 & & 3 & 0.5 \\
\hline 19 & 10.1 & 11.3 & \begin{tabular}{|l|}
12.4 \\
\end{tabular} & 13.4 & 14.3 & 15.1 & 15.9 & 16.5 & 17.1 & 17.7 & 18.2 & 18.7 & 19.1 & 19.5 & 19.9 & \begin{tabular}{|l|}
20.3 \\
\end{tabular} & 20.6 & 0.9 & 21.2 & 1.5 & 21.7 \\
\hline 20 & 10.6 & 11.9 & 13. & 14.2 & 15.1 & 16.0 & 16.7 & 17.4 & 18. & 18.7 & 19.4 & 19.7 & 20.2 & 20.6 & 21.0 & 21.4 & 21.1 & 2.1 & 22.4 & 22.7 & c.s \\
\hline 21 & 11.2 & 12.6 & \begin{tabular}{|l|l}
13.8 \\
\end{tabular} & 14.9 & 15. & 16.8 & 17.6 & - & 19. & 19.7 & 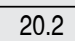 & 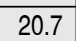 & 21.2 & 21.7 & 22.1 & \begin{tabular}{|l|}
22.5 \\
\end{tabular} & 22.9 & 23.2 & 23.5 & 23.8 & 241 \\
\hline 22 & 11.7 & 13.2 & \begin{tabular}{|l|l|}
14.5 \\
\end{tabular} & 15.7 & 16.7 & 17.7 & 18.5 & 19.3 & $\mid 20.0$ & 20.6 & 21.2 & 21.8 & 22.3 & 22.8 & 23.2 & \begin{tabular}{|l|}
23.6 \\
\end{tabular} & 4.0 & 4.4 & 24.7 & 25.0 & 25.3 \\
\hline 23 & 2.3 & \begin{tabular}{|l|l}
13.8 \\
\end{tabular} & \begin{tabular}{|l|}
15.2 \\
\end{tabular} & 16.4 & 17.5 & 18.5 & 19.4 & \begin{tabular}{|l}
20.2 \\
\end{tabular} & \begin{tabular}{|l|l}
20.9 \\
\end{tabular} & \begin{tabular}{|l|}
21.6 \\
\end{tabular} & 22.2 & 22 & 23.4 & 23.9 & \begin{tabular}{|l|}
24.3 \\
\end{tabular} & 24.8 & 5.2 & .5 & 9 & 26.2 & 26.5 \\
\hline 24 & 2.8 & $\begin{array}{ll}14.5 \\
\end{array}$ & 15.9 & 17.2 & 18.3 & 19.3 & 20.3 & 21 & 2 & 22.6 & 23 & 23 & 24.4 & 24.9 & \begin{tabular}{|l|}
25.4 \\
\end{tabular} & 5.9 & & .7 & .1 & .4 & 270 \\
\hline 25 & 3.4 & 15.1 & \begin{tabular}{|l|}
16.6 \\
\end{tabular} & 7.9 & 19.1 & 2 & 2 & \begin{tabular}{|l|l}
22.0 \\
\end{tabular} & \begin{tabular}{|l|l}
22.8 \\
\end{tabular} & \begin{tabular}{|l}
23.6 \\
\end{tabular} & 2 & 2 & 25.5 & 26.0 & & & & & & & 9.0 \\
\hline 26 & 1.0 & \begin{tabular}{|l|l}
15.7 \\
\end{tabular} & \begin{tabular}{|l|}
17.3 \\
\end{tabular} & 18.7 & 19.9 & 21.0 & 22.0 & \begin{tabular}{|l|l}
23.0 \\
\end{tabular} & \begin{tabular}{|l|l}
23.8 \\
\end{tabular} & 24.6 & 25.3 & 25.9 & 26. & 271 & 27.6 & 3.1 & 3.6 & 9.0 & 9.4 & 8 & 0.2 \\
\hline 27 & 4.5 & 16.4 & \begin{tabular}{|l|l|}
18.0 \\
\end{tabular} & 19.4 & 20.7 & 21. & 22.9 & 2 & 24 & 25.5 & 26 & 27 & 27.6 & 28.2 & 28. & 9.3 & 9.7 & .2 & 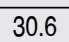 & .0 & 1.4 \\
\hline 28 & 15.1 & 17.0 & \begin{tabular}{|l|l}
18.7 \\
\end{tabular} & 20.2 & 21. & 22. & 23.8 & 2 & \begin{tabular}{|l|}
25.7 \\
\end{tabular} & 26 & & & 28.7 & 29.3 & 29. & 30.4 & 0.9 & . & 1.8 & 32.2 & 26 \\
\hline 29 & 15.6 & \begin{tabular}{|l}
17.6 \\
\end{tabular} & \begin{tabular}{|l|l}
19.3 \\
\end{tabular} & 0.9 & 22.3 & 23.5 & 24.7 & \begin{tabular}{|l|l}
25.7 \\
\end{tabular} & 26.6 & 27.5 & 28.3 & 29.0 & 29.7 & 30.4 & 31. & 31.5 & .0 & & & .4 & 3.8 \\
\hline 30 & 16.2 & \begin{tabular}{|l}
18.2 \\
\end{tabular} & 20.0 & 21.6 & 23.1 & 24. & 25.6 & 26 & 27 & 28.5 & 23 & 30 & 30 & 31 & 32. & 32. & 33.2 & .7 & 1 & 6 & 0 \\
\hline 31 & 16.8 & 18.9 & \begin{tabular}{|l|l}
20.7 \\
\end{tabular} & 22.4 & 23.9 & 25.2 & 26.4 & 27 & 28. & 29.5 & 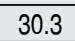 & & 31 & 32 & 33 & 33. & 34. & 34.8 & 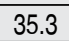 & 0 & 6.2 \\
\hline 32 & 17.3 & 19.5 & \begin{tabular}{|l|l}
21.4 \\
\end{tabular} & 23.1 & 24.7 & 26. & 2 & . & 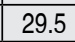 & 30 & & & 32 & 33.6 & 34.3 & & 35.4 & 0 & 6.5 & .0 & 7.4 \\
\hline 33 & 17.9 & 20.1 & \begin{tabular}{|l}
22.1 \\
\end{tabular} & & 25.5 & 2 & & & & 31 & & & 34 & 34 & & & & & & & \\
\hline 34 & 18.4 & 20.8 & \begin{tabular}{|l|l}
22.8 \\
\end{tabular} & 24. & 26.3 & 27.7 & 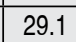 & 3 & 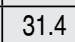 & 3 & & & 35 & 35. & 36 & 37 & 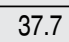 & 8.3 & 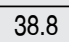 & 9.3 & \\
\hline 35 & 19.0 & 21.4 & \begin{tabular}{|l|}
23.5 \\
\end{tabular} & 25.4 & 27.1 & - & 30.0 & 3 & 3 & 33.4 & . .4 & .3 & 36.1 & & & & 38.9 & & 0.0 & 0.5 & - \\
\hline 36 & 19.5 & 22.0 & \begin{tabular}{|l|l}
24.2 \\
\end{tabular} & 26.1 & 27.9 & 29.4 & 30.8 & 3 & 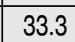 & 34.4 & 35.4 & 36.3 & 37.2 & 38.0 & 38.7 & \begin{tabular}{|l|}
39.4 \\
\end{tabular} & 40. & 6 & 1.2 & .7 & 42.2 \\
\hline 37 & & 22.6 & \begin{tabular}{|l|l}
24.9 \\
\end{tabular} & 26.9 & 28.7 & 30 & - & & & 3 & & 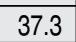 & 38 & & & 4 & & & & 9 & \\
\hline 38 & 20.7 & 23 & \begin{tabular}{|l}
25.6 \\
\end{tabular} & 27.6 & 29.5 & 31. & 32. & 34. & 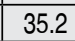 & 36.4 & 37.4 & 38.4 & 39 & 1 & 40 & 41. & 42 & 2.9 & 3.5 & 4.1 & \\
\hline 39 & 21.2 & 23.9 & \begin{tabular}{|l}
26.3 \\
\end{tabular} & 28.4 & 30.2 & 31. & 33.5 & 34.9 & 36.2 & 37.3 & 38.4 & 39.4 & 40 & 2 & 2.0 & 2.8 & 5 & 4.1 & .7 & 5.3 & 5.9 \\
\hline 40 & 21.8 & 4.5 & \begin{tabular}{|l|}
27.0 \\
\end{tabular} & 29.1 & 31.0 & 32. & 34.4 & & \begin{tabular}{|l|}
37. \\
\end{tabular} & 38 & 39.4 & 40.5 & 41. & & 43 & & & 3 & 9 & .5 & 47.1 \\
\hline 41 & 23 & \begin{tabular}{|l|l|}
252 \\
\end{tabular} & \begin{tabular}{|l|}
27.6 \\
\end{tabular} & 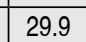 & 210 & & 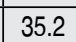 & 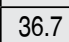 & 38 & 39 & 40 & 41 & 42 & 12 & 4 & 45. & 45.7 & 46.4 & - & 47 & 92 \\
\hline 42 & 22.9 & 25.8 & \begin{tabular}{|l|}
28.3 \\
\end{tabular} & .6 & 32.6 & & 36.1 & 0 & \begin{tabular}{|l|l}
39.0 \\
\end{tabular} & 40.3 & 47 & $4 c$ & 43 & 5 & 0.3 & 3.1 & 6.9 & 7.6 & 3.3 & 48.9 & 9.5 \\
\hline 43 & 23.5 & 26.4 & \begin{tabular}{|l|l}
29.0 \\
\end{tabular} & 31.4 & 33.4 & 35.3 & 37.0 & \begin{tabular}{|l}
38.6 \\
\end{tabular} & \begin{tabular}{|l|l}
40.0 \\
\end{tabular} & \begin{tabular}{|l|l}
41.3 \\
\end{tabular} & 42 & 43.6 & 44.6 & 45.5 & 46.4 & \begin{tabular}{|l|}
47.3 \\
\end{tabular} & 3.0 & 3.8 & 9.4 & 50.1 & 50.7 \\
\hline 44 & & 27. & 29 & 32. & & & 37 & & & & & & 45 & & & & & & & & 1.9 \\
\hline 45 & 24.6 & 27.7 & 30 & 32.8 & 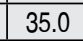 & & , & 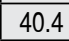 & 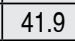 & 43 & 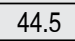 & 45 & 46 & 47 & 48. & 49.5 & 0.3 & .1 & 8 & .5 & 3.1 \\
\hline 46 & 25.1 & 28.3 & \begin{tabular}{|l|}
31.1 \\
\end{tabular} & 33.6 & 35.8 & 37.8 & 39.7 & 4 & 4 & 44.2 & 45 & 46.7 & 47 & 48.8 & 9.7 & 0.6 & 1.5 & 2.2 & 3.0 & 3.7 & 44.3 \\
\hline 47 & 25.7 & 28.9 & \begin{tabular}{|l|l}
31.8 \\
\end{tabular} & 34. & 36.6 & 38.7 & 40.5 & \begin{tabular}{|l|}
42. \\
\end{tabular} & \begin{tabular}{|l|}
43. \\
\end{tabular} & \begin{tabular}{|l}
45.2 \\
\end{tabular} & 46 & 47 & 48 & 49.9 & 50 & 51. & 52.6 & 53.4 & 54.1 & 54.8 & 5.5 \\
\hline 48 & 26.2 & 29.6 & \begin{tabular}{|l|}
32.5 \\
\end{tabular} & & 27 & $x_{1}$ & & & & 162 & & & 49 & & 52 & \begin{tabular}{|l|}
52 \\
\end{tabular} & $5 \pi$ & & 550 & 56.0 & 77 \\
\hline 49 & 26.8 & 30 & 33. & & & & & & & 4 & & & 51 & & & & 4.9 & 5.7 & 5 & 7.2 & 7.9 \\
\hline 50 & 27.4 & 30.8 & \begin{tabular}{|l|l}
33.9 \\
\end{tabular} & 36. & 39.0 & 41. & 43.2 & 45. & 46.6 & 48.2 & 49 & 50 & 52.0 & 1 & 54. & 55. & 56.0 & 9.9 & 7.7 & 8.4 & 9.1 \\
\hline 51 & 27. & 31. & 34.6 & 37. & 39.8 & 42. & 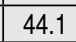 & 45 & 47 & 49.1 & 5 & & 53 & & 55 & 56 & 2 & 0 & 3.9 & 9.6 & .3 \\
\hline- & 28 & & \begin{tabular}{|l|}
35. \\
\end{tabular} & & & & & & & 201 & & & 54 & & 56 & 57 & 3 & 2 & 0 & 0.8 & -1 \\
\hline 53 & 29.0 & 32.7 & \begin{tabular}{|l}
35.9 \\
\end{tabular} & 38.8 & 41.4 & 43.7 & 45.8 & 4 & 4 & 51.1 & 5 & 53.9 & 55.2 & 4 & 57.5 & \begin{tabular}{|l|}
58.5 \\
\end{tabular} & 99.5 & 0.4 & 1.2 & 2.0 & 2.7 \\
\hline 54 & 29.6 & 33.3 & \begin{tabular}{|l|l}
36.6 \\
\end{tabular} & 39.6 & 42.2 & 44.6 & 46.7 & 48.7 & 50.4 & 52.1 & 53.6 & 55.0 & 56.3 & 57.5 & \begin{tabular}{|l|}
58.6 \\
\end{tabular} & \begin{tabular}{|l|}
59.6 \\
\end{tabular} & 60.6 & 1.5 & 62.4 & 3.2 & 64.0 \\
\hline 55 & 30.2 & 34 & \begin{tabular}{|l}
37.3 \\
\end{tabular} & 40.3 & 43.0 & 45.4 & & & & 53.1 & & 5 & 57 & & 59 & $=$ & 1 & 2.7 & & 4 & 5 \\
\hline 56 & 30 & & 38.0 & & 40 & & & & & & & & & & & & & & 4.7 & 5.6 & 5.4 \\
\hline 57 & 31.3 & 35.2 & \begin{tabular}{|l|l}
38.7 \\
\end{tabular} & 41.8 & 44.6 & 47.1 & 49.3 & 51.4 & 53.3 & 55.0 & 56.6 & 58.1 & 59.5 & 60.7 & \begin{tabular}{|l|}
61.9 \\
\end{tabular} & \begin{tabular}{|l|}
63.0 \\
\end{tabular} & 64.0 & 5.0 & 65.9 & 6.8 & 67.6 \\
\hline 58 & 31.8 & \begin{tabular}{|l|}
35.8 \\
\end{tabular} & \begin{tabular}{|l|}
39.4 \\
\end{tabular} & 42.5 & 45.4 & 47.9 & 50.2 & 52.3 & 54.3 & \begin{tabular}{|l|}
56.0 \\
\end{tabular} & 57.6 & 59.1 & 60.5 & 61.8 & 63.0 & \begin{tabular}{|l|}
64.1 \\
\end{tabular} & 65.2 & 6.2 & 67.1 & 68.0 & 68.8 \\
\hline 59 & 32.4 & 36.5 & \begin{tabular}{|l|l|}
$\mid$ & 40.1
\end{tabular} & 43. & 46.2 & 48.8 & 51. & 53. & 55.2 & \begin{tabular}{|l|}
57.0 \\
\end{tabular} & 58.6 & 60.2 & 61.6 & 62.9 & 64.1 & 5.3 & 6.3 & 7.3 & 68.3 & 69.2 & 70.0 \\
\hline 60 & 33.0 & 37.1 & 40.8 & 44. & 41. & 49. & 52.0 & \begin{tabular}{|l}
54.2 \\
\end{tabular} & 56.2 & 58.0 & 59.7 & 61.2 & 62.6 & 64.0 & 65.2 & 6.4 & 7.5 & 68.5 & 69.4 & 70.3 & 71.2 \\
\hline
\end{tabular}


Tabla 3: Valores predichos de DL,CO ajustados para carboxihemoglobina.

\begin{tabular}{|c|c|c|c|c|c|c|c|c|c|c|c|c|c|c|c|c|c|c|c|}
\hline \multirow{2}{*}{$\begin{array}{l}\text { DL, } \\
\text { co } \\
\% \mathrm{p}\end{array}$} & \multicolumn{19}{|c|}{ Valor de carboxihemoglobina ( $\mathrm{COHb}), \%$} \\
\hline & 2 & 3 & 4 & 5 & 6 & 7 & 8 & 9 & 10 & 11 & 12 & 13 & 14 & 15 & 16 & 17 & 18 & 19 & 20 \\
\hline 5 & 5.0 & 5.0 & 4.9 & 4.9 & 4.8 & 4.8 & 4.7 & 4.7 & 4.6 & 4.6 & 4.5 & 4.5 & 4.4 & 4.4 & 4.3 & 4.3 & 4.2 & 4.2 & 4.1 \\
\hline 6 & 6.0 & 5.9 & 5.9 & 5.8 & 5.8 & 5.7 & 5.6 & 5.6 & 5.5 & 5.5 & 5.4 & 5.3 & 5.3 & 5.2 & 5.2 & 5.1 & 5.0 & 5.0 & 4.9 \\
\hline 7 & 7.0 & 6.9 & 6.9 & 6.8 & 6.7 & 6.7 & 6.6 & 6.5 & 6.4 & 6.4 & 6.3 & 6.2 & 6.2 & 6.1 & 6.0 & 6.0 & 5.9 & 5.8 & 5.7 \\
\hline 8 & 8.0 & 7.9 & 7.8 & 7.8 & 7.7 & 7.6 & 7.5 & 7.4 & 7.4 & 7.3 & 7.2 & 7.1 & 7.0 & 7.0 & 6.9 & 6.8 & 6.7 & 6.6 & 6.6 \\
\hline 9 & 9.0 & 8.9 & 8.8 & 8.7 & 8.6 & 8.6 & 8.5 & 8.4 & 8.3 & 8.2 & 8.1 & 8.0 & 7.9 & 7.8 & 7.7 & 7.7 & 76 & 7.5 & 7.4 \\
\hline 10 & 10.0 & 9.9 & 9.8 & 9.7 & 9.6 & 9.5 & 9.4 & 9.3 & 9.2 & 9.1 & 9.0 & 8.9 & 8.8 & 8.7 & 8.6 & 8.5 & 8.4 & 8.3 & 8.2 \\
\hline 11 & 11.0 & 10.9 & 10.8 & 10.7 & 10.6 & 10.5 & 10.3 & 10.2 & 10.1 & 10.0 & 9.9 & 9.8 & 9.7 & 9.6 & 9.5 & 9.4 & 9.2 & 9.1 & 9.0 \\
\hline 12 & 12.0 & 11.9 & 11.8 & 11.6 & 11.5 & 11.4 & 11.3 & 11.2 & 11.0 & 10.9 & 10.8 & 10.7 & 10.6 & 10.4 & 10.3 & 10.2 & 10.1 & 10.0 & 9.8 \\
\hline 13 & 13.0 & 12.9 & 12.7 & 12.6 & 12.5 & 12.4 & 12.2 & 12.1 & 12.0 & 11.8 & 1.7 & 11.6 & 11.4 & 11.3 & 1.2 & 11.1 & .9 & 10.8 & 10.7 \\
\hline 14 & 14.0 & 13.9 & 13.7 & 13.6 & 13.4 & 13.3 & 13.2 & 13.0 & 12.9 & 12.7 & 12.6 & 12.5 & 12.3 & 12.2 & 12.0 & 11.9 & 11.8 & 11.6 & 11.5 \\
\hline 15 & 15.0 & 14.9 & 14.7 & 14.6 & 14.4 & 14.3 & 14.1 & 14.0 & 13.8 & 13.7 & 13.5 & 13.4 & 13.2 & 13.1 & 12.9 & 12.8 & 12.6 & 12.5 & 12.3 \\
\hline 16 & 16.0 & 15.8 & 15.7 & 15.5 & 15.4 & 15.2 & 15.0 & 14.9 & 14.7 & 14.6 & 14.4 & 14.2 & 14.1 & 13.9 & 13.8 & 13.6 & 13.4 & 13.3 & 13.1 \\
\hline 17 & 17.0 & 16.8 & 16.7 & 16.5 & 16.3 & 16.2 & 16.0 & 15.8 & 15.6 & 15.5 & 15.3 & 15.1 & 15.0 & 14.8 & & 14.5 & & 14.1 & 13.9 \\
\hline 18 & 18.0 & 17.8 & 17.6 & 17.5 & 17.3 & 17.1 & 16.9 & 16.7 & 16.6 & 16.4 & 16.2 & 16.0 & 15.8 & 15.7 & 5.5 & 15.3 & 5.1 & 14.9 & 14.8 \\
\hline 19 & 19.0 & 18.8 & 18.6 & 18.4 & 18.2 & 18.1 & 17.9 & 17.7 & 17.5 & 17.3 & 17.1 & 16.9 & 16.7 & 16.5 & 16.3 & 16.2 & 16.0 & 15.8 & 15.6 \\
\hline 20 & 20.0 & 19.8 & 19.6 & 19.4 & 19.2 & 19. & 18.8 & 18. & 18. & 18. & 18.0 & 17. & 17.6 & 17.4 & 17.2 & 17.0 & 16.8 & 16.6 & 16.4 \\
\hline 21 & 21.0 & 20.8 & 20.6 & 20.4 & 20.2 & 20. & 19.7 & 19. & 19. & 19 & 18. & 18.7 & 18. & 18.3 & 18.1 & 17.9 & 11 & 17.4 & 17.2 \\
\hline 22 & 22.0 & 21.8 & 21.6 & 21.3 & 21.1 & 20.9 & 20.7 & 20.5 & 20.2 & 20.0 & 19.8 & 19.6 & 19.4 & 19.1 & 18.9 & 18.7 & 18.5 & 18.3 & 18.0 \\
\hline 23 & 23.0 & 22.8 & 22.5 & 22.3 & 22.1 & 21.9 & 21.6 & 21.4 & 21.2 & 20.9 & 20.7 & 20.5 & 20.2 & 20.0 & 19. & 19.6 & 19.3 & 9.1 & 18.9 \\
\hline 24 & 24.0 & 23.8 & 23.5 & 23.3 & 23.0 & 22.8 & 22.6 & 22.3 & 22.1 & 21. & 21. & 21 & 21. & 20.9 & - & 20.4 & 20 & 19.9 & 19.7 \\
\hline 25 & 25.0 & 24.8 & 24.5 & 24.3 & 24.0 & 23.8 & 23.5 & 23.3 & 230 & 22. & $\sqrt{2}$ & 22.3 & 22.0 & 21.8 & & 21.3 & 0 & 20.8 & 20.5 \\
\hline 26 & 26.0 & 25.7 & 25.5 & 25.2 & 25.0 & 24.7 & 24.4 & 24.2 & 23.9 & 23. & 23.4 & 23.1 & 22.9 & 22.6 & 22.4 & 22.1 & 21.8 & 21.6 & 21.3 \\
\hline 27 & 27.0 & 26.7 & 26.5 & 26.2 & 25.9 & 25.7 & 25.4 & 25. & 24. & 24 & 24. & 24 & 23. & 23.5 & 23 & 23.0 & 22.7 & 22.4 & 22.1 \\
\hline 28 & 28.0 & 27.7 & 27.4 & 27.2 & 26.9 & 26.6 & 26.3 & 26.0 & & 25 & 25. & & 24.6 & 24.4 & 24.1 & 23.8 & 23.5 & 3.2 & 23.0 \\
\hline 29 & 29.0 & 28.7 & 28.4 & 28.1 & 27.8 & 27.6 & 27.3 & 27.0 & 267 & 26.4 & 26.1 & 25.8 & 25 & 25.2 & 24.9 & 24.7 & 24.4 & 24.1 & 23.8 \\
\hline 30 & 30.0 & 29.7 & 29.4 & 29.1 & 28.8 & 28. & 28.2 & 27 & 27.6 & 27 & 27. & 26.7 & 26. & 26.1 & $25 . \varepsilon$ & 25.5 & 25.2 & 24.9 & 24.6 \\
\hline 31 & 31.0 & 30.7 & 30.4 & 30.1 & 29.8 & 29. & 29.1 & 28. & 28.5 & 28. & 27.9 & 27 & 27. & 27.0 & 26. & 26.4 & 26. & 25.7 & 25.4 \\
\hline 32 & 32.0 & 31.7 & 31.4 & 31.0 & 30.7 & 30.4 & 30.1 & 29 & 29.4 & 29 & 28.8 & 28.5 & 28.2 & 27.8 & 27.5 & 27.2 & 9 & 26.6 & 26.2 \\
\hline 33 & 33.0 & 32.7 & 32.3 & 32.0 & 327 & 31. & 310 & 30 & P & 30 & 29.7 & 29.4 & 29. & 28.7 & 28.4 & 8.1 & 7 & 27.4 & 27.1 \\
\hline 34 & 34.0 & 33.7 & 33.3 & 33.0 & 32.6 & 32. & 32. & 31 & & 30 & 30. & 30.3 & 29. & 29.6 & 29. & 9 & 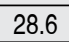 & 28.2 & 27.9 \\
\hline 35 & 35.0 & 34.7 & 34.3 & 34.0 & 33.6 & 33.3 & 32.9 & 32. & & & 31. & 0.2 & 30.8 & 30.5 & 30.1 & 9.8 & 29.4 & 9.1 & 28.7 \\
\hline 36 & 36.0 & 35.6 & 35.3 & 34.9 & 34.6 & 34.2 & 33.8 & 33.5 & 33.1 & 32.8 & 32.4 & 32.0 & 31. & 31.3 & 31. & 30.6 & 30.2 & 29.9 & 29.5 \\
\hline 37 & 37.0 & 36.6 & 36.3 & 35.9 & 35 & 35. & 210 & 34 & 31 & & & & & & & & & 7 & 3 \\
\hline 38 & 38.0 & 37.6 & 37.2 & 36.9 & 36.5 & 36. & 35.7 & 35. & 35. & 34. & 34. & 33. & 33. & 1 & 32.7 & 32.3 & 31.9 & 31.5 & 31.2 \\
\hline 39 & 39.0 & 38.6 & 38.2 & 37.8 & 37.4 & 37.1 & 36.7 & 36. & 35.9 & 35. & 35. & 34.7 & 34. & 33.9 & 33.5 & 33.2 & 32 & 32.4 & 32.0 \\
\hline 40 & 40.0 & 39.6 & 39.2 & 38.8 & 38.4 & 38 & 37.6 & 37. & 36.8 & & 36 & & 35 & & & 34.0 & & 33.2 & 32.8 \\
\hline 41 & 41.0 & 40.6 & 40.2 & 39.8 & 39.4 & 39. & 38 & 38 & 37 & 37 & 36 & 36 & 36 & 3 & 35 & 34.9 & 34. & 34.0 & 33.6 \\
\hline 42 & 42.0 & 41.6 & 41.2 & 40.7 & 40.3 & 39 & 39.5 & 39. & 38. & 38 & 37. & $3 / .4$ & 37. & 36.5 & 36.1 & 35.7 & 8 & 34.9 & 34.4 \\
\hline 43 & 43.0 & 42.6 & 42.1 & 41. & 41.3 & 40 & 40.4 & 40 & 39.6 & 39 & 38 & 38.3 & 37. & 37.4 & 37 & 36.6 & 36.1 & 35.7 & 35.3 \\
\hline 44 & 44.0 & 43. & & 42. & & 41 & 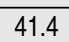 & 40 & . & & 39 & 302 & 38 & & & 37.4 & & 36.5 & 36.1 \\
\hline 45 & 45.0 & 44. & 44.1 & 43.7 & 43.2 & 42. & 42.3 & 41 & 41.4 & 41. & 40.5 & 40.1 & 39.6 & 39.2 & 38.7 & 38.3 & 37.8 & 37.4 & 36.9 \\
\hline 46 & 46.0 & 45.5 & 45.1 & 44.6 & 44.2 & 43.7 & 43.2 & 42.8 & 42.3 & 41.9 & 41.4 & 40.9 & 40.5 & 40.0 & 39.6 & 39.1 & 38.6 & 38.2 & 37.7 \\
\hline 47 & 47.0 & 46.5 & 46.1 & 45.6 & 45.1 & 44.7 & 44.2 & 43.7 & 43.2 & 42.8 & 42.3 & 41 & 41.4 & 40.9 & 40.4 & 40.0 & 39.5 & 39.0 & 38.5 \\
\hline 48 & 48.0 & 47.5 & 47.0 & 46.6 & 46.1 & 45. & 451 & 44. & 4 & 43. & 13 & 42 & 42 & 410 & 41 & 40.8 & 40 & 39.8 & 39.4 \\
\hline 49 & 49.0 & 48.5 & 48.0 & 47.5 & 47 & 46 & 46.1 & 45 & 45.1 & 44 & 44 & 43.6 & 4 & 42.6 & 1 & 41.7 & .2 & 40.7 & 40.2 \\
\hline 50 & 50.0 & 49.5 & 49.0 & 48.5 & 48.0 & 47.5 & 47.0 & 46 & 46.0 & 45.5 & 45.0 & 44.5 & 44.0 & 43.5 & 43.0 & 42.5 & 42.0 & 41.5 & 41.0 \\
\hline 51 & 51.0 & 50.5 & 50.0 & 49.5 & 49.0 & 48. & 47.9 & 47.4 & 46.9 & 46.4 & 45. & & 44. & 44.4 & 43 & 43.4 & 42.8 & 42.3 & 41.8 \\
\hline 52 & 52.0 & 51 & 51 & 50 & 49 & 49 & & & & & & & & & .7 & 44.2 & 3.7 & 3.2 & 42.6 \\
\hline 53 & 53.0 & 52.5 & 51.9 & 51.4 & 50.9 & 50.4 & 49.8 & 49.3 & 48.8 & 48.2 & 47.7 & 47.2 & 46.6 & 46.1 & 45.6 & 45.1 & 44.5 & 44.0 & 43.5 \\
\hline 54 & 54.0 & 53.5 & 52.9 & 52.4 & 51.8 & 51.3 & 50.8 & 50.2 & 49.7 & 49.1 & 48.6 & 48.1 & 47.5 & 47.0 & 46.4 & 45.9 & 45.4 & 44.8 & 44.3 \\
\hline 55 & 55. & 54. & 53. & 53. & 52 & 52. & 51. & 51 & 50. & & 49 & & 48.4 & 47.9 & 47.3 & 46.8 & 6.2 & 45.7 & 45.1 \\
\hline 56 & 56.0 & 55.4 & 54.9 & 54.3 & 53.8 & 53.2 & 52.6 & 52 & 51.5 & 51 & 50.4 & 49.8 & 49.3 & 48.7 & 48.2 & 47.6 & 47.0 & 46.5 & 45.9 \\
\hline 57 & 57.0 & 56.4 & 55.9 & 55.3 & 54.7 & 54.2 & 53.6 & 53.0 & 52.4 & 51.9 & 51.3 & 50.7 & 50.2 & 49.6 & 49.0 & 48.5 & 47.9 & 47.3 & 46.7 \\
\hline 58 & 58.0 & 57.4 & 56.8 & 56.3 & 55.7 & 55.1 & 54.5 & 53. & 53.4 & 52.8 & 52.2 & 51.6 & 51.0 & 50.5 & 49.9 & 49.3 & 48.7 & 48.1 & 47.6 \\
\hline 59 & 59.0 & 58.4 & 57.8 & 57.2 & 56.6 & 56.1 & 55.5 & 54. & 54.3 & 53.1 & 53.1 & 52.5 & 51.9 & 51.3 & 50.7 & 50.2 & 49.6 & 49.0 & 48.4 \\
\hline 60 & 60.0 & 59.4 & 58.8 & 58.2 & 57.6 & 57.0 & 56.4 & 55.8 & 55.2 & 54.6 & 54.0 & 53.4 & 52.8 & 52.2 & 51.6 & 51.0 & 50.4 & 49.8 & 49.2 \\
\hline
\end{tabular}


Vázquez-García JC et al. Prueba de difusión pulmonar de monóxido de carbono con técnica de una sola respiración

Tabla 4: Valores predichos de DL,CO ajustado para una altitud de $100 \mathrm{a} 2000 \mathrm{~m}$.

\begin{tabular}{|c|c|c|c|c|c|c|c|c|c|c|c|c|c|c|c|c|c|c|c|c|}
\hline \multirow{2}{*}{$\begin{array}{l}\text { DL, } \\
\text { CO } \\
\% \text { p }\end{array}$} & \multicolumn{20}{|c|}{ Altitud en metros } \\
\hline & 100 & 200 & 300 & 400 & 500 & 600 & 700 & 800 & 900 & 1000 & 1100 & 1200 & 1300 & 1400 & 1500 & \begin{tabular}{|l|}
1600 \\
\end{tabular} & 1700 & 1800 & 1900 & 2000 \\
\hline 5 & 5.0 & 5.1 & 5.1 & 5.1 & 5.1 & 5.3 & 5.2 & 5.2 & 5.3 & 5.3 & 5.3 & 5.3 & 5.4 & 5.4 & 5.4 & 5.5 & 5.5 & 5.5 & 5.6 & 5.6 \\
\hline 6 & 6.0 & 6.1 & 6.1 & 3.2 & 62 & 6.4 & 6.3 & 6.3 & 63 & 6.4 & 6.4 & 6.4 & 6.5 & 6.5 & 6.5 & 6.6 & 6.6 & 6.6 & 6.7 & 67 \\
\hline 7 & 7.0 & 7.1 & 7.1 & r & 7.2 & 7.5 & 7.3 & 7.3 & 7.4 & 7.4 & 7.5 & 7.5 & 7.5 & 7.6 & 7.6 & 7.7 & 7.7 & 7.7 & 7.8 & 7.8 \\
\hline 8 & 8.0 & 8.1 & 8.2 & 8.2 & 8.2 & 8.5 & 8.3 & 8.4 & 8.4 & 8.5 & 8.5 & 8.6 & 8.6 & 8.7 & 8.7 & 8.8 & 8.8 & 8.9 & 8.9 & 8.9 \\
\hline 9 & 9.1 & 9.1 & 9.2 & 9.2 & 9.3 & 9.6 & 9.4 & 9.4 & 9.5 & 9.5 & 9.6 & 9.6 & 9.7 & 9.8 & 9.8 & 9.9 & 9.9 & 10.0 & 10.0 & 10.1 \\
\hline 10 & 10.1 & 10.1 & 10.2 & 10.3 & 10.3 & 10.7 & \begin{tabular}{|l|l}
10.4 \\
\end{tabular} & 10.5 & 10.5 & 10.6 & 10.7 & 10.7 & 10.8 & 10.8 & 10.9 & \begin{tabular}{|l}
11.0 \\
\end{tabular} & 11.0 & 11.1 & 11.1 & 11.2 \\
\hline 11 & 11.1 & 11.1 & 11.2 & 11.3 & 11.3 & 11.7 & $\begin{array}{ll}11.5 \\
\end{array}$ & 11.5 & 11.6 & 11.7 & 11.7 & 11.8 & 11.8 & 11.9 & 12.0 & \begin{tabular}{|l}
12.0 \\
\end{tabular} & 12.1 & 12.2 & 12.2 & 12.3 \\
\hline 12 & 12.1 & \begin{tabular}{|l|}
12.2 \\
\end{tabular} & \begin{tabular}{|l}
12.2 \\
\end{tabular} & 12.3 & 12.3 & 12.8 & 12.5 & 12.6 & \begin{tabular}{|l}
12.6 \\
\end{tabular} & 12.7 & 12.8 & 12.8 & 12.9 & 13.0 & \begin{tabular}{|l|}
13.1 \\
\end{tabular} & \begin{tabular}{|l|}
13.1 \\
\end{tabular} & 13.2 & 13.3 & 13.3 & 13.4 \\
\hline 13 & 13.1 & 13.2 & 13.2 & 13.3 & 13.4 & 13.8 & 13.5 & 13.6 & 13.7 & 13.8 & 13.9 & 13.9 & 14.0 & 14.1 & \begin{tabular}{|l|}
14.1 \\
\end{tabular} & \begin{tabular}{|l}
14.2 \\
\end{tabular} & 14.3 & 14.4 & 14.4 & 14.5 \\
\hline 14 & 14.1 & 14.2 & 14.3 & 14.4 & 14.4 & 14.9 & 14.6 & 14.7 & 14.7 & 14.8 & 14.9 & 15.0 & 15.1 & 152 & 15.2 & \begin{tabular}{|l}
15.3 \\
\end{tabular} & 15.4 & 15.5 & 15.5 & 5.6 \\
\hline 15 & 15.1 & \begin{tabular}{|l}
15.2 \\
\end{tabular} & \begin{tabular}{|l}
15.3 \\
\end{tabular} & 15.4 & 15.4 & 16.0 & 15.6 & 15.7 & \begin{tabular}{|l|l}
15.8 \\
\end{tabular} & 15.9 & 16.0 & 16.0 & 16.2 & 16.3 & 16.3 & \begin{tabular}{|l|}
16.4 \\
\end{tabular} & 16.5 & 16.6 & 16.7 & 16.8 \\
\hline 16 & 16.1 & \begin{tabular}{|l|l}
16.2 \\
\end{tabular} & \begin{tabular}{|l|l}
16.3 \\
\end{tabular} & 16.4 & 16.5 & 17.0 & \begin{tabular}{|l|l}
16.7 \\
\end{tabular} & 16.8 & \begin{tabular}{|l|l}
16.8 \\
\end{tabular} & 16.9 & 17.1 & 17.1 & 17.2 & 17.3 & 17.4 & \begin{tabular}{|l|l}
17.5 \\
\end{tabular} & 17.6 & 17.7 & 17.8 & 17.9 \\
\hline 17 & 17.1 & \begin{tabular}{|l|}
17.2 \\
\end{tabular} & 17.3 & 17.4 & 17.5 & 18.1 & \begin{tabular}{|l|l}
17.7 \\
\end{tabular} & \begin{tabular}{ll|}
17.8 \\
\end{tabular} & \begin{tabular}{|l|l}
17.9 \\
\end{tabular} & 18.0 & $\begin{array}{ll}18.1 \\
\end{array}$ & 18.2 & 18.3 & 18.4 & \begin{tabular}{|l|}
18.5 \\
\end{tabular} & \begin{tabular}{|l|}
18.6 \\
\end{tabular} & 18.7 & 18.8 & 18.9 & 19.0 \\
\hline 18 & 18.1 & \begin{tabular}{|l}
18.2 \\
\end{tabular} & \begin{tabular}{|l|}
18.3 \\
\end{tabular} & 18.5 & 18.5 & 19.2 & \begin{tabular}{|l|l}
18.8 \\
\end{tabular} & \begin{tabular}{|l|l}
18.9 \\
\end{tabular} & 18.9 & 19.1 & 19.2 & 19.3 & \begin{tabular}{|l|l|}
19.4 \\
\end{tabular} & 19.5 & \begin{tabular}{|l|}
19.6 \\
\end{tabular} & \begin{tabular}{|l}
19.7 \\
\end{tabular} & \begin{tabular}{|l}
19.8 \\
\end{tabular} & 19.9 & 20.0 & 20.1 \\
\hline 19 & 19.1 & \begin{tabular}{|l|}
19.2 \\
\end{tabular} & \begin{tabular}{|l|l}
19.4 \\
\end{tabular} & 19.5 & 19.5 & 20.2 & \begin{tabular}{|l|l}
19.8 \\
\end{tabular} & 19.9 & \begin{tabular}{|l|l}
20.0 \\
\end{tabular} & 20.1 & 20.3 & 20.3 & \begin{tabular}{|l|}
20.5 \\
\end{tabular} & 20.6 & \begin{tabular}{|l|}
20.7 \\
\end{tabular} & \begin{tabular}{|l}
20.8 \\
\end{tabular} & \begin{tabular}{|l|}
20.9 \\
\end{tabular} & 21.0 & 21.1 & 21.2 \\
\hline 20 & 20.1 & \begin{tabular}{|l}
20.3 \\
\end{tabular} & 20.4 & 20.5 & 20.6 & 21.3 & 20.8 & 21.0 & \begin{tabular}{|l|l}
21.0 \\
\end{tabular} & 21. & 21.3 & 21. & \begin{tabular}{|l|l|}
21.5 \\
\end{tabular} & 21.7 & 21.8 & \begin{tabular}{|l|}
21.9 \\
\end{tabular} & 22.0 & 22.1 & 22.2 & 22.4 \\
\hline 21 & 21.1 & \begin{tabular}{|l}
21.3 \\
\end{tabular} & 21.4 & 21.5 & 21.6 & 22.4 & 21.9 & 22.0 & 22. & 22. & 22.4 & 22.5 & \begin{tabular}{|l|l}
22.6 \\
\end{tabular} & 22.8 & 22.8 & 23.0 & \begin{tabular}{|l|}
23.1 \\
\end{tabular} & 23.2 & 23.3 & 3.5 \\
\hline 22 & 22.1 & \begin{tabular}{|l}
22.3 \\
\end{tabular} & 22.4 & 22.6 & 22.6 & 23.4 & \begin{tabular}{|l}
22.9 \\
\end{tabular} & 23.1 & \begin{tabular}{|l|l|}
23.1 \\
\end{tabular} & 23.3 & 23.5 & 23.5 & \begin{tabular}{|l|l|}
23.7 \\
\end{tabular} & 3.8 & 3.9 & \begin{tabular}{|l|l|}
24.1 \\
\end{tabular} & \begin{tabular}{|l}
24.2 \\
\end{tabular} & 24.3 & 4.4 & 4.6 \\
\hline 23 & 23.1 & \begin{tabular}{|l|l}
23.3 \\
\end{tabular} & \begin{tabular}{|l|l}
23.4 \\
\end{tabular} & 23.6 & 23.7 & 24.5 & 24.0 & 24.1 & \begin{tabular}{|l}
24.2 \\
\end{tabular} & 24.4 & 24.5 & 24.6 & \begin{tabular}{|l|l}
24.8 \\
\end{tabular} & 24.9 & 25.0 & 25.2 & 25.3 & 25.4 & 25.5 & 25.7 \\
\hline 24 & 24.1 & \begin{tabular}{|l|l}
24.3 \\
\end{tabular} & \begin{tabular}{|l|l}
24.5 \\
\end{tabular} & 24.6 & 24.7 & 25.6 & 25.0 & 25.2 & 25. & 25. & 25.6 & 25. & \begin{tabular}{|l|l}
25.8 \\
\end{tabular} & 26.0 & 26.1 & \begin{tabular}{|l|}
26.3 \\
\end{tabular} & \begin{tabular}{|l|}
26.4 \\
\end{tabular} & 26.6 & 26.6 & 6.8 \\
\hline 25 & 25.2 & \begin{tabular}{|l|}
25.3 \\
\end{tabular} & \begin{tabular}{|l|}
25.5 \\
\end{tabular} & 25.6 & 25.7 & 26.6 & 26.0 & 26.2 & 26. & 26. & 26.7 & 26.7 & \begin{tabular}{|l|l}
26.9 \\
\end{tabular} & 7.1 & \begin{tabular}{|l|}
27.2 \\
\end{tabular} & \begin{tabular}{|l}
27.4 \\
\end{tabular} & \begin{tabular}{|l}
27.5 \\
\end{tabular} & 27.7 & 27.8 & .9 \\
\hline 26 & 26.2 & \begin{tabular}{|l|}
26.3 \\
\end{tabular} & \begin{tabular}{|l}
26.5 \\
\end{tabular} & 26.7 & 26.7 & 27.7 & \begin{tabular}{|l|l}
27.1 \\
\end{tabular} & 27.3 & \begin{tabular}{|l|l}
27.4 \\
\end{tabular} & 27.5 & 27.7 & 27.8 & \begin{tabular}{|l|l}
28.0 \\
\end{tabular} & 28.2 & \begin{tabular}{|l|}
28.3 \\
\end{tabular} & \begin{tabular}{|l}
28.5 \\
\end{tabular} & \begin{tabular}{|l}
28.6 \\
\end{tabular} & 28.8 & 28.9 & 9.1 \\
\hline 27 & 27.2 & \begin{tabular}{|l|l}
27.3 \\
\end{tabular} & 27.5 & 27.7 & 27.8 & 28.8 & 28.1 & 28.3 & \begin{tabular}{|l|l}
28.4 \\
\end{tabular} & 28. & 28 & 28. & 29.1 & 9.3 & 29.4 & 29.6 & 29.7 & 29.9 & 0 & 30.2 \\
\hline 28 & 28.2 & \begin{tabular}{|l|l}
28.4 \\
\end{tabular} & \begin{tabular}{|l}
28.5 \\
\end{tabular} & 28.7 & 28.8 & 29.8 & 29.2 & 29.4 & \begin{tabular}{|l}
29.5 \\
\end{tabular} & 29.7 & 29.9 & 29. & \begin{tabular}{|l|l}
30.1 \\
\end{tabular} & 30.4 & 30.5 & \begin{tabular}{|l}
30.7 \\
\end{tabular} & 30.8 & 31.0 & 31.1 & 31.3 \\
\hline 29 & 29.2 & 29.4 & 29.5 & 29.7 & 29.8 & 30.9 & 30.2 & 30.4 & \begin{tabular}{|l}
30.5 \\
\end{tabular} & 30.7 & 30.9 & 31.0 & 31.2 & 1.4 & 31.5 & \begin{tabular}{|l|}
31.8 \\
\end{tabular} & 31.9 & 32.1 & 32.2 & 4 \\
\hline 30 & 30.2 & \begin{tabular}{|l|l}
30.4 \\
\end{tabular} & 30.6 & 30.8 & 30.9 & 32.0 & 31.3 & 31. & 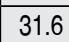 & 31. & 32 & 32 & 32. & 32.5 & 32. & \begin{tabular}{|l|}
32 \\
\end{tabular} & 33.0 & 33.2 & 3 & 5 \\
\hline 31 & 31.2 & \begin{tabular}{|l|l}
31.4 \\
\end{tabular} & \begin{tabular}{|l}
31.6 \\
\end{tabular} & 31.8 & 31.9 & 33.0 & 32. & 32. & \begin{tabular}{|l|}
32 \\
\end{tabular} & 32. & 33. & 33 & 33 & 33.6 & 33. & \begin{tabular}{|l}
33.9 \\
\end{tabular} & 34.1 & 34.3 & 34.4 & 4.7 \\
\hline 32 & 32.2 & \begin{tabular}{|l}
32.4 \\
\end{tabular} & \begin{tabular}{|l}
32.6 \\
\end{tabular} & 32.8 & 32.9 & 34.1 & 33. & 33.6 & 3 & 33. & 34 & 34 & 34 & 34.7 & 34. & \begin{tabular}{|l}
35.0 \\
\end{tabular} & \begin{tabular}{|l}
35.2 \\
\end{tabular} & 35.4 & 5.5 & 5.8 \\
\hline 33 & 33.2 & \begin{tabular}{|l|l}
33.4 \\
\end{tabular} & 6 & 33 & 3 & 35.1 & 34. & 34.6 & 3 & 35.0 & 35.2 & 35 & 35 & & & & 36.3 & 36.5 & & \\
\hline 34 & 34.2 & \begin{tabular}{|l|l}
34.4 \\
\end{tabular} & 34 & 34.9 & 35.0 & 36.2 & 35. & 35.7 & 35. & 36. & 36.2 & 36.4 & 36 & 36.9 & 37.0 & \begin{tabular}{|l}
37.2 \\
\end{tabular} & 37.4 & 37.6 & 37.7 & 0 \\
\hline 35 & 35.2 & \begin{tabular}{|l|l}
35.4 \\
\end{tabular} & \begin{tabular}{|l}
35.7 \\
\end{tabular} & 35 & 36 & 37.3 & 36.5 & 36.7 & \begin{tabular}{|l|}
36.8 \\
\end{tabular} & 37.1 & 37. & 37. & \begin{tabular}{|l}
37. \\
\end{tabular} & 37.9 & 38.1 & 8.3 & \begin{tabular}{|l}
38.5 \\
\end{tabular} & 38.7 & 38.9 & 39.1 \\
\hline 36 & 36.2 & \begin{tabular}{|l|}
36.5 \\
\end{tabular} & \begin{tabular}{|l}
36.7 \\
\end{tabular} & 36.9 & 37.0 & 38.3 & 37.5 & 37.8 & 37.9 & 38.1 & 38.4 & 38.5 & \begin{tabular}{|l|l}
38.8 \\
\end{tabular} & 39.0 & 39.2 & \begin{tabular}{|l|l}
39.4 \\
\end{tabular} & \begin{tabular}{|l|}
39.6 \\
\end{tabular} & 39.8 & 0.0 & 40.2 \\
\hline 37 & 37.2 & \begin{tabular}{|l|}
37.5 \\
\end{tabular} & \begin{tabular}{|l}
37.7 \\
\end{tabular} & 37.9 & 38 & 39.4 & 38.6 & 38.8 & 3 & 39.2 & 39.4 & 39 & 39 & & \begin{tabular}{|l|}
40.2 \\
\end{tabular} & 40 & \begin{tabular}{|l}
40.7 \\
\end{tabular} & 40.9 & .1 & \\
\hline 38 & 38.2 & \begin{tabular}{|l}
38.5 \\
\end{tabular} & \begin{tabular}{|l}
38.7 \\
\end{tabular} & 39.0 & 39.1 & 40.5 & 39. & 39.8 & 40. & 40. & 40.5 & 40 & \begin{tabular}{|l|l}
40.9 \\
\end{tabular} & 41.2 & 41.3 & \begin{tabular}{|l}
41. \\
\end{tabular} & 41.8 & 42.0 & 42.2 & 2.5 \\
\hline 39 & 39.2 & \begin{tabular}{|l|}
39.5 \\
\end{tabular} & \begin{tabular}{|l}
39.7 \\
\end{tabular} & 40.0 & 40.1 & 41.5 & 40.6 & 40.9 & \begin{tabular}{|l|}
41. \\
\end{tabular} & 41. & 41.6 & 41 & \begin{tabular}{|l}
42. \\
\end{tabular} & 42.3 & 42.4 & \begin{tabular}{|l}
42.7 \\
\end{tabular} & 42.9 & 43.1 & 43.3 & 3.6 \\
\hline 40 & 40.2 & \begin{tabular}{|l|l}
40.5 \\
\end{tabular} & 40.8 & 41.0 & 41.1 & 42. & 41. & 42.0 & \begin{tabular}{|l}
42. \\
\end{tabular} & 42.4 & 42.6 & 42.8 & \begin{tabular}{|l|l}
43.1 \\
\end{tabular} & 43.4 & \begin{tabular}{|l|l}
43.5 \\
\end{tabular} & \begin{tabular}{|l}
43.8 \\
\end{tabular} & 44.0 & 44.3 & 4 & \begin{tabular}{|l|l}
44.7 \\
\end{tabular} \\
\hline 41 & 41.3 & \begin{tabular}{|l|}
41.5 \\
\end{tabular} & & 42 & 42.2 & 43.7 & 42 & 43. & \begin{tabular}{|l|}
43 \\
\end{tabular} & 13 & 43. & 43 & \begin{tabular}{|l|}
44 \\
\end{tabular} & 44.4 & 44. & \begin{tabular}{|l|l}
44.9 \\
\end{tabular} & 45.0 & 45.4 & .5 & 8 \\
\hline 42 & 42.3 & 42. & \begin{tabular}{|l}
42.8 \\
\end{tabular} & 43 & 43.2 & 44.7 & 43. & 44.0 & \begin{tabular}{|l}
44.2 \\
\end{tabular} & 44.5 & 44.8 & 44.9 & \begin{tabular}{|l|}
45.2 \\
\end{tabular} & 45.5 & 45.7 & \begin{tabular}{|l|l}
46.0 \\
\end{tabular} & 46.1 & 46.5 & 46.6 & 46.9 \\
\hline 43 & 43.3 & \begin{tabular}{|l}
43.5 \\
\end{tabular} & 43.8 & 44.1 & 44.2 & 45.8 & \begin{tabular}{|l}
44.8 \\
\end{tabular} & 45.1 & \begin{tabular}{|l|l}
45.2 \\
\end{tabular} & 45.5 & 45.8 & 46.0 & \begin{tabular}{|l|l} 
\\
\end{tabular} 6.3 & 46.6 & 46.8 & \begin{tabular}{|l|}
47.1 \\
\end{tabular} & 47.2 & 47.6 & 7.7 & 48.1 \\
\hline 44 & 44.3 & \begin{tabular}{|l|l}
44.6 \\
\end{tabular} & 4 & 45 & 45 & 46 & 45 & 46 & 46 & 46 & 46.9 & 47.1 & \begin{tabular}{|l|l}
47.4 \\
\end{tabular} & $4 I$ & 47. & \begin{tabular}{|l}
48.2 \\
\end{tabular} & 48.3 & 7 & 8 & 2 \\
\hline 45 & 45.3 & \begin{tabular}{|l|}
45.6 \\
\end{tabular} & 4 & 46 & 46 & 479 & 46. & 47.2 & \begin{tabular}{|l}
47. \\
\end{tabular} & 47 & 48 & 48 & \begin{tabular}{|l|l|}
48.5 \\
\end{tabular} & 48.8 & 48. & \begin{tabular}{|l}
49.3 \\
\end{tabular} & 49.4 & 49.8 & .0 & 3 \\
\hline 46 & 46.3 & \begin{tabular}{|l}
46.6 \\
\end{tabular} & 46.9 & 47.2 & 47 & 49.0 & \begin{tabular}{|l}
47.9 \\
\end{tabular} & 48.2 & \begin{tabular}{|l|l}
48.4 \\
\end{tabular} & 48.7 & 49.0 & 49.2 & \begin{tabular}{|l|l}
49.5 \\
\end{tabular} & 49.9 & 50.0 & \begin{tabular}{|l}
50.4 \\
\end{tabular} & 50.5 & 50.9 & 1.1 & 51.4 \\
\hline 47 & 47.3 & \begin{tabular}{|l}
47.6 \\
\end{tabular} & 47.9 & 48.2 & 48.3 & 50.1 & 49.0 & 49.3 & \begin{tabular}{|l}
49.5 \\
\end{tabular} & 49.8 & 50.1 & 50.3 & \begin{tabular}{|l|l}
50.6 \\
\end{tabular} & 50.9 & 51. & \begin{tabular}{|l}
51.5 \\
\end{tabular} & 51.6 & 52.0 & 52.2 & 52.5 \\
\hline 48 & 48.3 & \begin{tabular}{|l|}
48. \\
\end{tabular} & & $4 \varsigma$ & 49 & 51.1 & 50. & 50. & 50 & 50. & 51. & 51 & 51 & 52.0 & 52.2 & \begin{tabular}{|l|}
52.6 \\
\end{tabular} & 52.7 & 53.1 & 53.3 & 53.7 \\
\hline 49 & 49.3 & \begin{tabular}{|l}
49 \\
\end{tabular} & 4 & - & 5 & 52 & 51. & 51 & 51 & 51. & 52 & 52.4 & \begin{tabular}{|l|}
52 \\
\end{tabular} & 53.1 & 53.3 & \begin{tabular}{|l}
53.7 \\
\end{tabular} & \begin{tabular}{|l}
53.8 \\
\end{tabular} & 54.2 & 4 & 8 \\
\hline 50 & 50.3 & \begin{tabular}{|l|l}
50.6 \\
\end{tabular} & 50.9 & 51.3 & 51.4 & 53.3 & \begin{tabular}{|l}
52.1 \\
\end{tabular} & 52.4 & \begin{tabular}{|l|l}
52.6 \\
\end{tabular} & 53.0 & 53.3 & 53.5 & \begin{tabular}{|l|l}
53.8 \\
\end{tabular} & 54.2 & 54.4 & \begin{tabular}{|l}
54.8 \\
\end{tabular} & \begin{tabular}{|l}
54.9 \\
\end{tabular} & 55.3 & 5.5 & 5.9 \\
\hline 51 & 51.3 & \begin{tabular}{|l|}
51.6 \\
\end{tabular} & \begin{tabular}{|l}
52.0 \\
\end{tabular} & 52 & 52 & 54.3 & 53.1 & 53.5 & \begin{tabular}{|l}
53.7 \\
\end{tabular} & 54.0 & 54.4 & 54.6 & \begin{tabular}{|l}
54.9 \\
\end{tabular} & 55.3 & \begin{tabular}{|l|}
55.5 \\
\end{tabular} & \begin{tabular}{|l}
55.8 \\
\end{tabular} & 56.0 & 56.4 & 56.6 & 57.0 \\
\hline 52 & 52.3 & \begin{tabular}{|l|}
52. \\
\end{tabular} & & & & & 54. & 54.5 & \begin{tabular}{|l|}
54 \\
\end{tabular} & 55. & 55.4 & 55. & \begin{tabular}{|l|l}
56.0 \\
\end{tabular} & 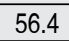 & 56 & \begin{tabular}{|l|}
56.9 \\
\end{tabular} & \begin{tabular}{|l|}
57.1 \\
\end{tabular} & 57.5 & 57.7 & 58.1 \\
\hline 53 & 53.3 & \begin{tabular}{|l}
53.7 \\
\end{tabular} & 54.0 & 54.3 & 5 & 56.4 & 55.2 & 55.6 & \begin{tabular}{|l|l}
55.8 \\
\end{tabular} & 56.1 & 56.5 & 56.7 & \begin{tabular}{|l|l}
57.1 \\
\end{tabular} & .5 & \begin{tabular}{|l|}
57.6 \\
\end{tabular} & \begin{tabular}{|l}
58.0 \\
\end{tabular} & 58.2 & 58.6 & 58.8 & 59.2 \\
\hline 54 & 54.3 & \begin{tabular}{|l}
54.7 \\
\end{tabular} & 55.0 & 55.4 & 55.5 & 57.5 & 56.3 & 56.6 & \begin{tabular}{|l|l}
56.8 \\
\end{tabular} & 57.2 & 57.6 & 57.8 & \begin{tabular}{|l|l}
58.1 \\
\end{tabular} & 58.5 & \begin{tabular}{|l|l}
58.7 \\
\end{tabular} & \begin{tabular}{|l|}
59.1 \\
\end{tabular} & \begin{tabular}{|l|}
59.3 \\
\end{tabular} & 59.7 & 59.9 & 60.4 \\
\hline 55 & 55.3 & \begin{tabular}{|l|l}
55.7 \\
\end{tabular} & \begin{tabular}{|l}
56.0 \\
\end{tabular} & 56.4 & 56.6 & 58.6 & \begin{tabular}{|l}
57.3 \\
\end{tabular} & 57.7 & \begin{tabular}{|l}
57.9 \\
\end{tabular} & 58.3 & 58.6 & 58.8 & \begin{tabular}{|l}
59.2 \\
\end{tabular} & 59.6 & 59.8 & \begin{tabular}{|l}
60.2 \\
\end{tabular} & \begin{tabular}{|l}
60.4 \\
\end{tabular} & 60.8 & 61.1 & .5 \\
\hline 56 & 56.3 & \begin{tabular}{|l|}
56.7 \\
\end{tabular} & 5 & & & & 58.4 & & & 59.3 & 59.7 & 500 & & & 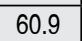 & G13 & \begin{tabular}{|l}
61.5 \\
\end{tabular} & 62.0 & 62.2 & 6 \\
\hline 57 & 57.4 & \begin{tabular}{|l|l}
57.7 \\
\end{tabular} & \begin{tabular}{|l|l}
58.1 \\
\end{tabular} & 58.4 & 58.6 & 60.7 & 59.4 & 59.8 & \begin{tabular}{|l|l}
60.0 \\
\end{tabular} & 60.4 & 60.8 & 61.0 & \begin{tabular}{|l|l|}
61.4 \\
\end{tabular} & 61.8 & \begin{tabular}{|l|}
62.0 \\
\end{tabular} & \begin{tabular}{|l|}
62.4 \\
\end{tabular} & \begin{tabular}{|l}
62.6 \\
\end{tabular} & 63.1 & 63.3 & $\begin{array}{ll}63.7 \\
\end{array}$ \\
\hline 58 & 58.4 & \begin{tabular}{|l|l}
58.7 \\
\end{tabular} & \begin{tabular}{|l|}
59.1 \\
\end{tabular} & 59.5 & 59.7 & 61.8 & 60.4 & 60.8 & \begin{tabular}{|l|l}
61.0 \\
\end{tabular} & 61.4 & 61.8 & 62.0 & \begin{tabular}{|l|l|}
62.5 \\
\end{tabular} & 62.9 & 63.1 & \begin{tabular}{|l}
63.5 \\
\end{tabular} & \begin{tabular}{|l}
63.7 \\
\end{tabular} & 64.2 & 64.4 & 64.8 \\
\hline 59 & 59.4 & \begin{tabular}{|l}
59.7 \\
\end{tabular} & \begin{tabular}{|l|l}
60.1 \\
\end{tabular} & 60.5 & 60.7 & 62.8 & $\begin{array}{l}61.5 \\
\end{array}$ & 61.0 & \begin{tabular}{|l|l}
62.1 \\
\end{tabular} & 62.5 & 62.9 & 63.1 & \begin{tabular}{|l|l|}
63.5 \\
\end{tabular} & 64.0 & 64.2 & \begin{tabular}{|l|l}
64.6 \\
\end{tabular} & \begin{tabular}{|l|}
64.8 \\
\end{tabular} & 65.3 & 65.5 & 66.0 \\
\hline 60 & 60.4 & 60.8 & \begin{tabular}{|l}
61.1 \\
\end{tabular} & 61.5 & 61.7 & 63.9 & 62.5 & 62.9 & \begin{tabular}{|l|l}
63.1 \\
\end{tabular} & 63.5 & 64.0 & 64.2 & \begin{tabular}{|l|l|}
64.6 \\
\end{tabular} & 65.0 & 65.3 & 65.7 & 65.9 & 66.4 & 66.6 & 67.1 \\
\hline
\end{tabular}


Tabla 5: Valores predichos de DL,CO ajustado para una altitud de (2100 a $4000 \mathrm{~m}$ ).

\begin{tabular}{|c|c|c|c|c|c|c|c|c|c|c|c|c|c|c|c|c|c|c|c|c|}
\hline \multirow{2}{*}{$\begin{array}{l}\text { DL, } \\
\text { CO } \\
\% \mathrm{p}\end{array}$} & \multicolumn{20}{|c|}{ Altitud en metros } \\
\hline & 100 & 200 & 2300 & 2400 & 2500 & 600 & 2700 & 2800 & 2900 & 3000 & 3100 & 3200 & 3300 & 100 & 500 & 600 & 700 & 3800 & 3900 & 4000 \\
\hline 5 & 5.6 & 5.6 & 5.7 & 5.7 & 5.7 & 5.7 & 5.8 & 5.8 & 5.8 & 5.9 & 5.9 & 5.9 & 6.0 & 6.0 & 5.5 & 6.0 & 6.1 & 6.1 & 6.1 & 6.1 \\
\hline 6 & 7 & - & & S & 6.9 & 69 & 6.9 & 7.0 & 70 & & 71 & & & 72 & 60 & 7.2 & 73 & 2 & - & 74 \\
\hline 7 & 7.9 & 7.9 & 7.9 & 8.0 & 8.0 & 8.0 & 8.1 & 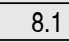 & 8.2 & 8.2 & 8.3 & 8.3 & 8.3 & 8.4 & 7.7 & 8.4 & 8.5 & 8.5 & 8.6 & 8.6 \\
\hline 8 & 9.0 & 9.0 & 9.1 & 9.1 & 9.2 & 9.2 & 9.3 & 9.3 & 9.3 & 9.4 & 9.4 & 9.5 & 9.5 & 9.6 & 8.8 & 9.6 & 9.7 & 9.8 & 9.8 & 9.8 \\
\hline 9 & $\begin{array}{l}0.1 \\
\end{array}$ & 10.2 & 10.2 & 10.3 & 10.3 & 10.3 & 10.4 & 10.5 & 10.5 & 10.6 & 10.6 & 10.7 & 10.7 & $\begin{array}{l}10.8 \\
\end{array}$ & 9.9 & 10.8 & 10.9 & 111.0 & 11.0 & 11.1 \\
\hline 10 & 11.2 & 11.3 & 11.3 & 11.4 & 11.5 & $4-5$ & 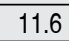 & 4 & 11.7 & 11.7 & 11.8 & & 110 & 12.0 & 11.0 & 12.1 & 121 & 12.2 & & 23 \\
\hline 11 & 12.3 & 12.4 & 12.5 & 12.6 & 12.6 & 12.6 & 12.7 & 12.8 & 12.8 & 12.9 & 13.0 & 13.0 & 13.1 & 3.2 & 12.1 & \begin{tabular}{|l}
13.3 \\
\end{tabular} & 13.4 & 3.4 & 3.5 & 3.5 \\
\hline 12 & 13.5 & 13.6 & 13.6 & 13.7 & 13.7 & 13.8 & 13.9 & 13.9 & 14.0 & 14.1 & 14.1 & 14.2 & 14.3 & 4.4 & 13.2 & 14.5 & 14.6 & 4.6 & 4.7 & \begin{tabular}{|l}
14.7 \\
\end{tabular} \\
\hline 13 & 14.6 & 14.7 & 14.7 & 14.8 & 14.9 & 14.9 & 15.1 & 15.1 & 15.2 & 15 & 15.3 & 4 & 5.5 & & 14.3 & 15.7 & 15.8 & 8.8 & 9 & 6.0 \\
\hline 14 & 15.7 & 15.8 & 15.9 & 16.0 & 16.0 & 16.1 & 62 & 163 & 16.3 & 16. & 16.5 & 56 & 16.7 & 168 & 154 & \begin{tabular}{|l|}
16.9 \\
\end{tabular} & 70 & 7.1 & 7.1 & 7.2 \\
\hline 15 & $\begin{array}{l}16.8 \\
\end{array}$ & 16.9 & 17.0 & 17.1 & 17.2 & 17.2 & 17.4 & 17.4 & 17.5 & \begin{tabular}{|l|}
17.6 \\
\end{tabular} & 17.7 & 17.8 & 7.9 & & 6.5 & 18.1 & 18.2 & 3.3 & 8.4 & 8.4 \\
\hline 16 & 17.9 & 18.1 & 18.1 & 18.3 & 18.3 & 18.4 & $2-$ & 10 & 18.7 & 18 & 1 & 3.9 & 9.1 & 19. & 17.6 & 19.3 & 19.4 & 9.5 & 19.6 & 9.7 \\
\hline 17 & 19.1 & 19.2 & 19.3 & 19.4 & 19 & & & & 19.8 & & & & & & & \begin{tabular}{|l}
20.5 \\
\end{tabular} & & & & \\
\hline 18 & \begin{tabular}{|l}
20.2 \\
\end{tabular} & 20.3 & 20.4 & 20.5 & 20.6 & 20.7 & 20 & 2 & 21.0 & 21 & 2 & & 1.5 & & & \begin{tabular}{|l}
21.7 \\
\end{tabular} & & & & 2.1 \\
\hline 19 & \begin{tabular}{|l}
21.3 \\
\end{tabular} & 21.5 & 21.5 & 21.7 & 21.8 & 21.8 & 22.0 & 22.1 & 22.2 & \begin{tabular}{|l|}
22 \\
\end{tabular} & 22 & 22 & 2.7 & $\angle L$ & 20.9 & 22.9 & 1 & 2 & 3 & 23.3 \\
\hline 20 & 22.4 & 22.6 & 22.7 & 22.8 & 22.9 & 23. & 23 & 23 & 23.3 & 23 & 23.6 & 2.1 & 23.8 & 23. & 22.0 & \begin{tabular}{|l|}
24.1 \\
\end{tabular} & 4.3 & .4 & 24.5 & 24.6 \\
\hline 21 & 23.6 & 23.7 & 23.8 & 24.0 & 24.1 & 24 & & & 24.5 & & & & 0 & & 23 & \begin{tabular}{|l|}
25.3 \\
\end{tabular} & & & & 50 \\
\hline 22 & 24.7 & 24.9 & 24.9 & 25.1 & 25.2 & 25.3 & 25.5 & 25.6 & 25.7 & 25 & 25 & 26.0 & 26.2 & 26. & 24. & 26. & 26.7 & & .9 & 7.0 \\
\hline 23 & 25.8 & 26.0 & 26.1 & 26.3 & 26.3 & 26. & 26. & & 26.8 & 27 & 27 & 27 & 27.4 & 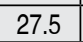 & & 27.7 & $2 t$ & 0 & 1 & \\
\hline 24 & 26. & \begin{tabular}{|l|}
27.1 \\
\end{tabular} & 27.2 & 27.4 & 27.5 & 27. & 27.8 & & 28.0 & 28 & & 28 & 28.6 & 28.7 & & \begin{tabular}{|l|}
28.9 \\
\end{tabular} & 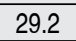 & 3 & & \\
\hline 25 & 28.0 & \begin{tabular}{|l}
28.2 \\
\end{tabular} & 28.3 & 28.5 & 28.6 & 28.7 & 28.9 & \begin{tabular}{|l|}
$x^{2}$ \\
\end{tabular} & 29.2 & 20 & & & & & & & & & & \\
\hline 26 & 9.2 & \begin{tabular}{|l|}
29.4 \\
\end{tabular} & 29.5 & 29.7 & 29.8 & 29. & 30.1 & 3 & 30.3 & 30 & - & ת & 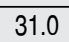 & 1. & 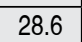 & 31.3 & & 7 & & .9 \\
\hline 27 & 30. & \begin{tabular}{|l}
30.5 \\
\end{tabular} & 30.6 & 30.8 & 30. & & 31 & & 31 & & & & 2.2 & & & |320 & & 2.9 & & 2.2 \\
\hline 28 & 31 & 31 & 31 & 32. & 32.1 & 32 & 32 & & 36 & & & & & & & & & & & \\
\hline 29 & 325 & \begin{tabular}{|l}
32.8 \\
\end{tabular} & 32.9 & 33.1 & 33.2 & 33.3 & 33.6 & 3 & 338 & 34.1 & 3 & 34 & 4.6 & 34.7 & & & & & & 5.6 \\
\hline 30 & 33. & 33. & 34.0 & 34.2 & 34.4 & 34 & 34 & & 35 & & & & 5.8 & 35. & & & & & & .9 \\
\hline 31 & 34. & 35. & 35. & 35. & 35. & 35 & 35 & 36 & 36 & 36 & & 36 & 37. & 37 & & 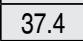 & & 37.8 & .9 & 31 \\
\hline 32 & 35. & & 36 & 36. & 36.7 & 36 & 37 & & 37 & & & 31 & 38.1 & & & & & & 39.2 & 3 \\
\hline 33 & (27) & & & 37. & 37. & 37. & & & & & & & & & & & & & & .5 \\
\hline 34 & 38. & 38 & 38. & 38.8 & 39. & 39 & 30 & & 39 & & & & 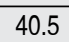 & 40. & & & & 1.5 & & .8 \\
\hline 35 & 39.3 & 36 & 39.7 & 40.0 & 40.1 & 40 & & & 40.8 & & & & & & & & & $\therefore .1$ & & 3.0 \\
\hline 36 & \begin{tabular}{|l}
40.4 \\
\end{tabular} & 40.7 & 40.8 & 41.1 & 41.2 & 41.4 & 44.7 & & 42.0 & 42 & 42 & 42 & 2.9 & 43. & & 4 & 7 & 9 & 4.1 & 4.2 \\
\hline 37 & 4 & & 41. & 42. & 42. & & & & & & & & & & & & & & & -2 \\
\hline 38 & 42 & 42. & 43 & 43. & 43. & 43 & & & 44 & & 44 & 4 & 45. & 45 & & 5.8 & 5 & 3 & .5 & 46.7 \\
\hline 39 & 43. & 44 & 44 & 44. & 44.7 & 44 & 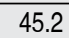 & & 45 & & & - & 6.5 & & & .0 & 4 & 5 & .7 & 7.9 \\
\hline 40 & \begin{tabular}{|l|}
44.9 \\
\end{tabular} & & & 45. & 45 & 46 & & & & & & & & & & & & & & - \\
\hline 41 & 460 & 46 & 16 & 46. & 47 & 47 & & & 47 & 48 & 48 & - & 18 & - & & 9.4 & & 50.0 & 0.2 & 0.4 \\
\hline 42 & 477.1 & 47 & 47 & 47.9 & 48.1 & 48 & 48 & & 49.0 & & $4 \varepsilon$ & 40.1 & .1 & & & 0.6 & .0 & .2 & .4 & 1.6 \\
\hline 43 & 48.2 & \begin{tabular}{|l}
48.6 \\
\end{tabular} & 48.7 & 49.1 & 49.3 & 49.4 & 49.8 & \begin{tabular}{|l}
50.0 \\
\end{tabular} & 50.2 & 50. & 50.7 & 50.9 & 51.3 & 51. & 47.2 & 51.8 & 52.2 & .4 & 6 & 2.8 \\
\hline 44 & & & & & 50 & & & & & & & & & & & & & & & \\
\hline 45 & 50. & \begin{tabular}{|l|}
50. \\
\end{tabular} & & 5 & 0 & 51 & 52 & & 52 & & & & 3.6 & 53.8 & & 54.2 & 1.7 & .9 & 5.1 & 5.3 \\
\hline 46 & \begin{tabular}{|l|l}
51.6 \\
\end{tabular} & \begin{tabular}{|l}
52.0 \\
\end{tabular} & 52.1 & 52.5 & 52.7 & 52.9 & 53.3 & 53.5 & \begin{tabular}{|l|l}
53.7 \\
\end{tabular} & 54.0 & 54.2 & 54 & 54.8 & 55.0 & 0.5 & \begin{tabular}{|l}
55.5 \\
\end{tabular} & 5.9 & 3.1 & 6.3 & 56.5 \\
\hline 47 & \begin{tabular}{|l}
52.7 \\
\end{tabular} & \begin{tabular}{|l|}
53. \\
\end{tabular} & 53.3 & 53.7 & 53.8 & 54 & 54. & 54 & 54 & & & & 50 & 56. & & \begin{tabular}{|l|}
56.7 \\
\end{tabular} & 1 & 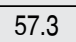 & & 57.7 \\
\hline 48 & -8 & 54 & & 548 & 55. & & 55 & & 56 & 56 & & & 57 & 57 & & 570 & & & 7 & 500 \\
\hline 49 & 55 & 55 & & 55 & 56 & & & & & & & & & & & 9.1 & & .7 & 0 & .2 \\
\hline 50 & \begin{tabular}{|l|}
56.1 \\
\end{tabular} & 56.5 & 56. & 57.1 & 57.3 & 57.5 & 57.9 & 58. & 58.3 & 58 & $5 \varsigma$ & 59.2 & 59.6 & 59. & 54. & 3 & .7 & .0 & 1.2 & 61.4 \\
\hline 51 & \begin{tabular}{|l|}
57. \\
\end{tabular} & & & $5 \varepsilon$ & 58 & & 59 & & & & & & & & & & & 2 & 2.4 & 2.7 \\
\hline 52 & & & & & & & & & & & & & & & & & & & & 3.9 \\
\hline 53 & \begin{tabular}{|l}
59.5 \\
\end{tabular} & 59.9 & 60.1 & 60.5 & 60.7 & 60.9 & 61.4 & 6 & \begin{tabular}{|l|l|}
61.8 \\
\end{tabular} & 62.3 & 62.5 & 62.7 & 63.2 & 63.4 & 58.2 & \begin{tabular}{|l|l}
63.9 \\
\end{tabular} & 1.4 & .6 & 4.9 & 55.1 \\
\hline 54 & \begin{tabular}{|l}
60.6 \\
\end{tabular} & 61.0 & 61.2 & 61.6 & 61.9 & 62.1 & 62. & \begin{tabular}{|l|}
62 \\
\end{tabular} & 63.0 & 63. & 63.7 & 63.9 & 64.4 & 64.6 & \begin{tabular}{|l|}
59.3 \\
\end{tabular} & \begin{tabular}{|l}
65.1 \\
\end{tabular} & 5.6 & 5.8 & 6.1 & 66.3 \\
\hline 55 & $\begin{array}{l}61 . \\
\end{array}$ & & 62. & 62. & 63. & 63 & & & & & & 65. & & & 60.4 & 3 & & .1 & 7.3 & 77.6 \\
\hline 56 & \begin{tabular}{|l|}
62. \\
\end{tabular} & 63 & 63 & 63 & 64 & & & & & & & & & & & & 0 & & 3.5 & 3.8 \\
\hline 57 & \begin{tabular}{|l|}
63.9 \\
\end{tabular} & 64.4 & 64.6 & 65.1 & 65.3 & 65.5 & 66.0 & \begin{tabular}{|l|l}
66.2 \\
\end{tabular} & 66.5 & 67.0 & 67.2 & 67.5 & 68.0 & 68.2 & 62.6 & \begin{tabular}{|l}
68.7 \\
\end{tabular} & 69.2 & 9.5 & 69.8 & 70.0 \\
\hline 58 & 65. & 65 & 65.7 & 66. & 66.4 & 66.7 & 67. & 67 & 67. & 68 & $6 \varepsilon$ & 68 & 69.1 & 69. & 63 & $v_{4}$ & 4 & 0.7 & 1.0 & 71.3 \\
\hline 59 & 66. & 66.6 & 66. & $6 \%$. & 67.6 & 67. & 60 & & 68. & $6 y$ & $0 y$ & 09. & 70.3 & 10.0 & 64. & 1.1 & $71 .{ }^{\prime}$ & 71.9 & 2.2 & 2.5 \\
\hline 60 & 67.3 & 67.8 & 68.0 & 68.5 & 68.7 & 69.0 & 69.5 & 69.1 & 70.0 & 70.5 & / 0.1 & 71.0 & 71.5 & 71.8 & 65.9 & 72.3 & 72.9 & 73.2 & 73.4 & 3.7 \\
\hline
\end{tabular}


Tabla 6: Criterios para graduación de calidad de la prueba de DL,CO.

\begin{tabular}{|c|c|c|c|c|c|c|}
\hline Grado & $\mathbf{V}_{\mathbb{I N}},(\%)$ & TA $(\mathbf{s e g})$ & $\mathbf{V}_{\mathbf{D}}(\mathbf{L})$ & $\mathbf{V}_{\mathbf{s}}(\mathbf{m L})$ & Repetibilidad & $\begin{array}{c}\text { Espirogramas } \\
\text { aceptable }\end{array}$ \\
\hline 1 & $\geq 90$ & $9-11$ & $0.50-1.00$ & $0.50-1.00$ & $\leq 1.0$ & 2 \\
\hline 2 & $85-89$ & $9-11$ & $0.50-1.00$ & $0.50-1.00$ & $1.1-1.9$ & 2 \\
\hline 3 & $85-89$ & $8-12$ & $0.50-1.00$ & $0.50-1.00$ & $2.0-2.9$ & 2 \\
\hline 4 & $80-84$ & $<80>12$ & $<0.500>1.10$ & $<0.500>1.10$ & $3.0-3.9$ & 2 \\
\hline 5 & $<80$ & $<80>12$ & $<0.500>1.10$ & $<0.500>1.10$ & $\geq 4.0$ & 2 \\
\hline 6 & $<80$ & $<80>12$ & $<0.500>1.10$ & $<0.500>1.10$ & $\geq 4.0$ & 1 \\
\hline 7 & & & & & & 0 \\
\hline
\end{tabular}

\section{Proceso de interpretación básica}

1. Graduación de calidad: aunque no hay un sistema de graduación de la calidad para la prueba de $\mathrm{DL}, \mathrm{CO}$, se propone una graduación de acuerdo con los estándares de aceptabilidad y repetibilidad (tabla 6).

2. La elección de la ecuación de referencia puede afectar la interpretación final. Cada laboratorio debe seleccionar la ecuación más apropiada para los métodos y la población seleccionada. Esto es esencial ya que se han descrito grandes diferencias entre las ecuaciones de referencia. ${ }^{15,28}$

3. Para la interpretación deben considerarse los valores ajustados pertinentes para la altitud $\left(\mathrm{PiO}_{2}\right)$, el valor de $\mathrm{Hb}$ y $\mathrm{COHb}$ (tablas 1 a 5).

4. Definir el patrón de transferencia de CO:

a. DL,CO normal, entre límites inferior y superior normal (cerca de entre 80 y $120 \%$ ).

b. DL,CO disminuida, menor al límite inferior normal $(<80 \%)$.

c. DL,CO aumentada, superior al límite superior normal (> 120\%).

d. Los valores limítrofes (75 a 80\%) deben interpretarse con precaución.

5. Graduación de la gravedad:
a. Disminución leve: DL,CO $60 \%$ al LIN.
b. Disminución moderada: DL,CO 40-59\%.
c. Disminución grave: $\mathrm{DL}, \mathrm{CO}<40 \%$.

\section{REFERENCIAS}

1. Crapo RO, Forster RE 2nd. Carbon monoxide diffusing capacity. Clin Chest Med 1989;10(2):187-198.

2. Krogh M. The diffusion of gases through the lungs of man. J Physiol 1915;49(4):271-300.
3. Macintyre N, Crapo RO, Viegi G, et al:; American Thoracic Society/ European Respiratory Society. Standardization of the single breath determination of carbon monoxide uptake in the lung. Eur Respir $\mathrm{J}$ 2005;26(4):720-735.

4. Wanger J, Clausen JL, Coates A, et al.; American Thoracic Society/ European Respiratory Society. Standardisation of the measurement of lung volumes. Eur Respir J 2005;26(3):511-522.

5. Jensen RL, Crapo RO. Diffusing capacity: how to get it right. Respir Care 2003;48(8):777-782.

6. Epler GR, Saber FA, Gaensler EA. Determination of severe impairment (disability) in interstitial lung disease. Am Rev Respir Dis 1980;121(4):647-659.

7. Nordenfelt I, Svensson G. The transfer factor (diffusing capacity) as a predictor of hypoxaemia during exercise in restrictive and chronic obstructive pulmonary disease. Clin Physiol 1987;7(5):423-430.

8. Gelb AF, Gold WM, Wright RR, Bruch HR, Nadel JA. Physiologic diagnosis of subclinical emphysema. Am Rev Respir Dis 1973;107(1):50-63.

9. Renzetti $A D$, Bleecker ER, Epler GR, et al. Evaluation of impairment/ disability secondary to respiratory disorders. Statement of the American Thoracic Society. Am Rev Respir Dis 1986;133(6):12051209.

10. Owens GR, Rogers RM, Pennock BE, Levin D. The diffusing capacity as a predictor of arterial oxygen desaturation during exercise in patients with chronic obstructive pulmonary disease. N Engl J Med 1984;310(19):1218-1221.

11. Mohsenifar Z, Brown HV, Schnitzer B, Prause JA, Koerner SK. The effect of abnormal levels of hematocrit on the single breath diffusing capacity. Lung 1982;160(6):325-330.

12. Clark EH, Woods RL, Hughes JM. Effects of blood transfusion on the carbon monoxide transfer factor of the lung in man. Clin Sci Mol Med 1978;54(6):627-631.

13. Cotes JE, Dabbs JM, Elwood PC, Hall AM, McDonald A, Saunders MJ. Iron-deficiency anaemia: its effect on transfer factor for the lung (diffusing capacity) and ventilation and cardiac frequency during submaximal exercise. Clin Sci 1972;42(3):325-335.

14. Cotes JE. Lung function. 5th Ed. London: Blackwell Scientific Publications; 1993.

15. Mottram CD. Ruppel's manual of pulmonary function testing. 10th ed. Maryland Heights: Elsevier; 2013.

16. Miller MR, Hankinson J, Brusasco V, et al.; ATS/ERS Task Force. Standardisation of spirometry. Eur Respir J 2005;26(2):319-338. 
17. Jones RS, Meade F. A theoretical and experimental analysis of anomalies in the estimation of pulmonary diffusing capacity by the single breath method. Q J Exp Physiol Cogn Med Sci 1961;46:131-143.

18. Morris AH, Crapo RO. Standardization of computation of single-breath transfer factor. Bull Eur Physiopathol Respir 1985;21(2):183-189.

19. Gray C, Zamel N, Crapo RO. Effect of a simulated 3,048 meter altitude on the single-breath transfer factor. Bull Eur Physiopathol Respir 1986;22(5):429-431.

20. Stam H, Kreuzer FJ, Versprille A. Effect of lung volume and positional changes on pulmonary diffusing capacity and its components. J Appl Physiol (1985) 1991;71(4):1477-1488.

21. Milne JA, Mills RJ, Coutts JRT, Macnaughton MC, Moran F, Pack Al. The effect of human pregnancy on the pulmonary transfer factor for carbon monoxide as measured by the single breath method. Clin Sci Mol Med 1977;53(3):271-276.

22. Gazioglu K, Kaltreider NL, Rosen M, Yu PN. Pulmonary function during pregnancy in normal women and in patients with cardiopulmonary disease. Thorax 1970;25(4):445-450.
23. Krumholz RA, Echt CR, Ross JC. Pulmonary diffusing capacity, capillary blood volume, lung volumes, and mechanics of ventilation in early and late pregnancy. J Lab Clin Med 1964;63:648-655.

24. Frey TM, Crapo RO, Jensen RL, Elliott CG. Diurnal variation of the diffusing capacity of the lung: is it real? Am Rev Respir Dis 1987;136(6):1381-1384.

25. Sansores RH, Abboud RT, Kennell C, Haynes N. The effect of menstruation on the pulmonary carbon monoxide diffusing capacity. Am J Respir Crit Care Med 1995;152(1):381-384.

26. Peavy HH, Summer WR, Gurtner $\mathrm{C}$. The effects of acute ethanol ingestion on pulmonary diffusing capacity. Chest 1980;77(4):488-492.

27. Marrades RM, Diaz O, Roca J, etal. Adjustment of DLCO for hemoglobin concentration. Am J Respir Crit Care Med 1997;155(1):236-241.

28. McGrath MW, Thomson ML. The effect of age, body size and lung volume change on alveolar-capillary permeability and diffusing capacity in man. J Physiol 1959;146(3):572-582.

Los autores declaran no tener conflicto de intereses. 